Pure and Applied Mathematics Quarterly

Volume 3, Number 2

(Special Issue: In honor of

Leon Simon, Part 1 of 2)

$513-538,2007$

\title{
Maps of Bounded Variation with Values into a Manifold: Total Variation and Relaxed Energy
}

\author{
Mariano Giaquinta and Domenico Mucci
}

In this paper we illustrate some ideas and results concerning the following question which is relevant in several instances and, in particular, in the calculus of variations. Given an integral energy $\mathcal{E}(u)$, as for instance the Dirichlet energy, the total variation or the area, and a sequence of smooth maps $u_{k}$ from the unit ball $B^{n}$ of $\mathbb{R}^{n}$ into an oriented, compact and boundaryless smooth Riemannian manifold $\mathcal{Y}$ in such a way that $\sup _{k} \mathcal{E}\left(u_{k}\right)<\infty$, we would like to describe the limit points of $u_{k}$. As it has been shown particularly in the last fifteen years and is quite well known, the process of convergence of the $u_{k}$ 's in general produces concentration of energy and loss of geometrical information. Our problem is that of recovering as much as possible of the energy and of the geometry of the limit points. As shown in [9] one way of doing this is by looking at the graphs of the $u_{k}$ 's, i.e., in terms of Cartesian currents: this allows at least to preserve the homological properties of the maps $u_{k}$ in the limit. In this paper we focus on and survey some recent results concerning the case in which $\mathcal{E}(u)$ is the total variation of $u$, and our main reference will be, besides [9], the recent paper [12].

\section{The TOTAL VARIATION}

In the sequel $B^{n}$ will denote the unit ball in $\mathbb{R}^{n}$ and $\mathcal{Y}$ a smooth oriented Riemannian manifold of dimension $M \geq 1$, isometrically embedded in $\mathbb{R}^{N}$ for some $N \geq 2$. We shall assume that $\mathcal{Y}$ is compact, connected, without boundary. In addition, we assume that its integral 1 -homology group $H_{1}(\mathcal{Y}):=H_{1}(\mathcal{Y} ; \mathbb{Z})$ has no torsion.

Vector VAlued $B V$-FunCtions. Let $u: B^{n} \rightarrow \mathbb{R}^{N}$ be a function in $B V\left(B^{n}, \mathbb{R}^{N}\right)$, i.e., $u=\left(u^{1}, \ldots u^{N}\right)$ with all components $u^{j} \in B V\left(B^{n}\right)$. We recall that the Jump set of $u$ is the countably $\mathcal{H}^{n-1}$-rectifiable set $J_{u}$ in $B^{n}$ given

Received November 29, 2005. 
by the union of the complements of the Lebesgue sets of the $u^{j}$ 's. Let $\nu=\nu_{u}(x)$ be a unit vector in $\mathbb{R}^{n}$ orthogonal to $J_{u}$, that exists at $\mathcal{H}^{n-1}$-a.e. point $x \in J_{u}$, and let $u^{ \pm}(x)$ denote the one-sided approximate limits of $u$ on $J_{u}$, so that for $\mathcal{H}^{n-1}$-a.e. point $x \in J_{u}$

$$
\lim _{\rho \rightarrow 0^{+}} \rho^{-n} \int_{B_{\rho}^{ \pm}(x)}\left|u(x)-u^{ \pm}(x)\right| d x=0,
$$

where $B_{\rho}^{ \pm}(x):=\left\{y \in B_{\rho}(x): \pm\langle y-x, \nu(x)\rangle \geq 0\right\}$. Note that a change of sign of $\nu$ induces a permutation of $u^{+}$and $u^{-}$and that only for scalar functions there is a canonical choice of the sign of $\nu$ which ensures that $u^{+}(x)>u^{-}(x)$. The distributional derivative of $u$ decomposes into the sum of a "gradient" measure, which is absolutely continuous with respect to the Lebesgue measure, of a "jump" measure, concentrated on a set that is $\sigma$-finite with respect to the $\mathcal{H}^{n-1}$-measure, and of a "Cantor-type" measure. More precisely,

$$
D u=D^{a} u+D^{J} u+D^{C} u
$$

where

$$
D^{a} u=\nabla u \cdot d x, \quad D^{J} u=\left(u^{+}(x)-u^{-}(x)\right) \otimes \nu(x) \mathcal{H}^{n-1}\left\llcorner J_{u},\right.
$$

$\nabla u:=\left(\nabla_{1} u, \ldots, \nabla_{n} u\right)$ being the approximate gradient of $u$, compare e.g. [6], [16], [2], [9, Vol. I]. We also recall that $\left\{u_{k}\right\}$ is said to converge to $u$ weakly in the $B V$-sense, $u_{k} \rightarrow u$, if $u_{k} \rightarrow u$ strongly in $L^{1}\left(B^{n}, \mathbb{R}^{N}\right)$ and $D u_{k} \rightarrow D u$ weakly in the sense of (vector-valued) measures. We will finally set

$$
B V\left(B^{n}, \mathcal{Y}\right):=\left\{u \in B V\left(B^{n}, \mathbb{R}^{N}\right) \mid u(x) \in \mathcal{Y} \text { for } \mathcal{L}^{n} \text {-a.e. } x \in B^{n}\right\} .
$$

The tOtal VARIATion. To every map $u \in B V\left(B^{n}, \mathcal{Y}\right)$ we associate its total variation, given for every Borel set $B \subset B^{n}$ by

$$
\mathcal{E}_{T V}(u, B):=\int_{B}|\nabla u(x)| d x+\left|D^{C} u\right|(B)+\int_{J_{u} \cap B} \mathcal{H}^{1}\left(l_{x}\right) d \mathcal{H}^{n-1}(x) .
$$

Here, for any $x \in J_{u}$, we let $\mathcal{H}^{1}\left(l_{x}\right)$ denote the length of a geodesic arc $l_{x}$ in $\mathcal{Y}$ with initial and final points $u^{-}(x)$ and $u^{+}(x)$. Moreover we set

$$
\mathcal{E}_{T V}(u):=\mathcal{E}_{T V}\left(u, B^{n}\right) .
$$

Note that if $u$ is smooth, at least in $W^{1,1}\left(B^{n}, \mathcal{Y}\right)$, i.e. $u \in W^{1,1}\left(B^{n}, \mathbb{R}^{N}\right)$ with $u(x) \in \mathcal{Y}$ for $\mathcal{L}^{n}$-a.e. $x \in B^{n}$, then

$$
\mathcal{E}_{T V}(u, B)=\int_{B}|D u| d x .
$$

Moreover, clearly for every $u \in B V\left(B^{n}, \mathcal{Y}\right)$ we have

$$
|D u|(B) \leq \mathcal{E}_{T V}(u, B) .
$$

LOWER SEMICONTINUITY. By slicing it is then not difficult to show the following. 
Proposition 1.1. Let $n \geq 2$ and $u \in B V\left(B^{n}, \mathcal{Y}\right)$. For every sequence of smooth maps $\left\{u_{k}\right\} \subset C^{1}\left(B^{n}, \mathcal{Y}\right)$ such that $u_{k} \rightarrow u$ weakly in the $B V$-sense, and for any open set $A \subset B^{n}$, we have

$$
\liminf _{k \rightarrow \infty} \int_{A}\left|D u_{k}\right| d x \geq \mathcal{E}_{T V}(u, A) .
$$

DENSITY RESULTS. The previous definition of the total variation is motivated by the 1 -dimensional case, $n=1$. In fact, we have

Theorem 1.2. For every $u \in B V\left(B^{1}, \mathcal{Y}\right)$ there exists a sequence of smooth maps $\left\{u_{k}\right\} \subset C^{1}\left(B^{1}, \mathcal{Y}\right)$ such that $u_{k} \rightarrow u$ weakly in the $B V$-sense and $\left\|D u_{k}\right\|_{L^{1}} \rightarrow$ $\mathcal{E}_{T V}(u)$ as $k \rightarrow \infty$.

If $n \geq 2$, this is not anymore true. Indeed, the map $x /|x|$ from $B^{2}$ into $S^{1}$ has no smooth strict approximation as in Theorem 1.2. In fact, were this true, according to what we are going to discuss below, the currents $G_{u_{k}}$ integration over the graphs of the $u_{k}$ 's would converge to the current integration over the graph of $x /|x|$, thus the boundary $\partial G_{x /|x|}\left\llcorner B^{2} \times S^{1}\right.$ would be zero, since by Stokes theorem $\partial G_{u_{k}}\left\llcorner B^{2} \times S^{1}=0\right.$ for every $k$, while $\partial G_{x /|x|}\left\llcorner B^{2} \times S^{1}=\right.$ $-\delta_{0} \times \llbracket S^{1} \rrbracket$, as it is easily seen.

However, it turns out that the density results of Bethuel [3] in the setting of the Sobolev space $W^{1,1}\left(B^{n}, \mathcal{Y}\right)$ extend to the class $B V\left(B^{n}, \mathcal{Y}\right)$. Recall that $R_{1}^{\infty}\left(B^{n}, \mathcal{Y}\right)$ denotes the set of all the maps $u \in W^{1,1}\left(B^{n}, \mathcal{Y}\right)$ which are smooth except on a singular set $\Sigma(u)$ of the type

$$
\Sigma(u)=\bigcup_{i=1}^{r} \Sigma_{i}, \quad r \in \mathbb{N},
$$

where $\Sigma_{i}$ is a smooth $(n-2)$-dimensional subset of $B^{n}$ with smooth boundary, if $n \geq 3$, and $\Sigma_{i}$ is a point if $n=2$. The following density results appear in [3].

Theorem 1.3. The class $R_{1}^{\infty}\left(B^{n}, \mathcal{Y}\right)$ is strongly dense in $W^{1,1}\left(B^{n}, \mathcal{Y}\right)$.

Theorem 1.4. The class $C^{1}\left(B^{n}, \mathcal{Y}\right)$ is dense in $R_{1}^{\infty}\left(B^{n}, \mathcal{Y}\right)$ in the strong $W^{1,1}$ topology if and only if the first homotopy group of $\mathcal{Y}$ is trivial, i.e., $\pi_{1}(\mathcal{Y})=0$.

The following holds for maps in $B V\left(B^{n}, \mathcal{Y}\right)$, see [12].

Theorem 1.5. Let $n \geq 2$. For every $u \in B V\left(B^{n}, \mathcal{Y}\right)$ there exists a sequence of maps $\left\{u_{k}\right\} \subset R_{1}^{\infty}\left(B^{n}, \mathcal{Y}\right)$ such that $u_{k} \rightarrow u$ as $k \rightarrow \infty$ weakly in the $B V$-sense and

$$
\lim _{k \rightarrow \infty} \int_{B^{n}}\left|D u_{k}\right| d x=\mathcal{E}_{T V}\left(u, B^{n}\right) .
$$

As a consequence, by using Theorem 1.4 we immediately infer the following. 
Corollary 1.6. Suppose that $\pi_{1}(\mathcal{Y})=0$. For every $u \in B V\left(B^{n}, \mathcal{Y}\right)$ there exists a sequence of smooth maps $\left\{u_{k}\right\} \subset C^{1}\left(B^{n}, \mathcal{Y}\right)$ such that $u_{k} \rightarrow u$ as $k \rightarrow \infty$ weakly in the $B V$-sense and (1.2) holds true.

ThE RELAXED $B V$-ENERGY. In view of the above it is reasonable to study the lower semicontinuous envelope of the functional $\overline{\mathcal{E}_{T V}}: B V\left(B^{n}, \mathcal{Y}\right) \rightarrow[0,+\infty]$ given by

$$
\overline{\mathcal{E}_{T V}}(u):= \begin{cases}\int_{B^{n}}|D u| d x & \text { if } u \in C^{1}\left(B^{n}, \mathcal{Y}\right) \\ +\infty & \text { elsewhere in } B V\left(B^{n}, \mathcal{Y}\right) .\end{cases}
$$

More precisely, we define the relaxed $B V$-energy $\widetilde{\mathcal{E}_{T V}}: B V\left(B^{n}, \mathcal{Y}\right) \rightarrow[0,+\infty]$ as the greatest functional on $B V\left(B^{n}, \mathcal{Y}\right)$ which is lower than or equal to $u \mapsto$ $\overline{\mathcal{E}_{T V}}(u)$ and is lower semicontinuous with respect to the weak convergence in the $B V$-sense.

By Proposition 1.1 we clearly infer that

$$
\mathcal{E}_{T V}(u) \leq \widetilde{\mathcal{E}_{T V}}(u) \quad \forall u \in B V\left(B^{n}, \mathcal{Y}\right)
$$

Therefore, by Corollary 1.6 we readily obtain

Corollary 1.7. If $\pi_{1}(\mathcal{Y})=0$, then equality holds in (1.4) for every $u \in B V\left(B^{n}, \mathcal{Y}\right)$.

However, if $\pi_{1}(\mathcal{Y}) \neq 0$, in general the strict inequality " $<"$ may hold in (1.4), even for Sobolev maps $u \in W^{1,1}\left(B^{n}, \mathcal{Y}\right)$, as we have seen.

In order to analyze the structure property of the relaxed $B V$-energy, first we introduce the class of Cartesian currents cart $^{1,1}\left(B^{n} \times \mathcal{Y}\right)$ which arise in a natural way as weak limits of sequences of graphs of smooth maps $u_{k}: B^{n} \rightarrow \mathcal{Y}$ with equibounded $W^{1,1}$-norms. We first focus on the easier case of dimension $n=1$, where the main features can be easily outlined.

CURRENTS CARRIED BY $B V$-FUnCTIONS. The structure of a function in $B V\left(B^{n}, \mathcal{Y}\right)$ suggests to associate to any such function $u$ a suitable current $T_{u} \in$ $\mathcal{D}_{n, 1}\left(B^{n} \times \mathcal{Y}\right)$, i.e., a linear continuous functional acting on compactly supported smooth $n$-forms in $\mathcal{D}^{n}\left(B^{n} \times \mathcal{Y}\right)$ with at most one vertical differential, compare Sec. 3 below. Any form $\omega \in \mathcal{D}^{n, 1}\left(B^{n} \times \mathcal{Y}\right)$ may be decomposed as $\omega=\omega^{(0)}+\omega^{(1)}$ according to the number of vertical components, where

$$
\omega^{(0)}=\phi(x, y) d x \quad \text { and } \quad \omega^{(1)}=\sum_{j=1}^{N} \sum_{i=1}^{n}(-1)^{n-i} \phi_{i}^{j}(x, y) \widehat{d x^{i}} \wedge d y^{j}
$$

for some $\phi \in C_{c}^{\infty}\left(B^{n} \times \mathcal{Y}\right)$ and $\phi_{i}^{j} \in C_{0}^{\infty}\left(B^{n} \times \mathcal{Y}\right)$, and

$$
\widehat{d x^{i}}:=d x^{1} \wedge \cdots d x^{i-1} \wedge d x^{i-1} \wedge \cdots \wedge d x^{n} .
$$


We split

$$
T_{u}:=T_{u}^{a}+T_{u}^{C}+T_{u}^{J}
$$

into its absolutely continuous, Cantor and Jump parts, and define $T_{u}\left(\omega^{(0)}\right)=$ $T_{u}^{a}\left(\omega^{(0)}\right)$, where

$$
T_{u}^{a}(\phi(x, y) d x):=\int_{B^{n}} \phi(x, u(x)) d x \quad \forall \phi \in C_{c}^{\infty}\left(B^{n} \times \mathcal{Y}\right),
$$

so that $T_{u}^{J}\left(\omega^{(0)}\right)=T_{u}^{C}\left(\omega^{(0)}\right)=0$. Moreover, if $\omega=\omega^{(1)}$ satisfies (1.5), we set

$$
\begin{aligned}
T_{u}^{a}\left(\omega^{(1)}\right) & :=\sum_{j=1}^{N} \int_{B^{n}}\left\langle\nabla u^{j}(x), \phi^{j}(x, u(x))\right\rangle d x \\
T_{u}^{C}\left(\omega^{(1)}\right) & :=\sum_{j=1}^{N} \int_{B^{n}} \phi^{j}(x, u(x)) d D^{C} u^{j} \\
T_{u}^{a}\left(\omega^{(1)}\right) & :=\sum_{j=1}^{N} \sum_{i=1}^{n} \int_{J_{u}}\left(\int_{\gamma_{x}} \phi_{i}^{j}(x, y) d y^{j}\right) \nu_{i} d \mathcal{H}^{n-1}(x) .
\end{aligned}
$$

Here $\phi^{j}:=\left(\phi_{1}^{j}, \ldots, \phi_{n}^{j}\right)$ and, for any $x \in J_{u}, \gamma_{x}$ agrees with the 1-current integration on an oriented geodesic arc $l_{x}$ in $\mathcal{Y}$ with initial and final points respectively given by $u^{-}(x)$ and $u^{+}(x)$, so that $\partial \llbracket l_{x} \rrbracket=\delta_{u^{+}(x)}-\delta_{u^{-}(x)}$.

We notice that the definition of $T_{u}$ depends on the choice of the geodesic arcs $l_{x}$. In particular, if $u \in W^{1,1}\left(B^{n}, \mathcal{Y}\right)$, clearly $T_{u}=T_{u}^{a}$ and hence $T_{u}$ agrees with the current $G_{u}$ integration of forms in $\mathcal{D}^{n, 1}\left(B^{n} \times \mathcal{Y}\right)$ over the rectifiable graph of $u$, defined in an approximate sense by

$$
G_{u}:=(I d \bowtie u)_{\#} \llbracket B^{n} \rrbracket,
$$

where $(I d \bowtie u)(x):=(x, u(x))$, compare [9].

Moreover, by Sec. 3 below we infer that the parametric variational integral $\mathcal{F}_{1,1}$ associated to the total variation integral satisfies for every Borel set $B \subset B^{n}$

$$
\mathcal{F}_{1,1}\left(T_{u}, B \times \mathcal{Y}\right)=\mathcal{E}_{T V}(u, B) \quad \forall u \in B V\left(B^{n}, \mathcal{Y}\right) .
$$

Also, in any dimension $n \geq 2$ we have

Proposition 1.8. For every $u \in B V\left(B^{n}, \mathcal{Y}\right)$ we find the existence of a sequence of maps $\left\{u_{k}\right\} \subset R_{1}^{\infty}\left(B^{n}, \mathcal{Y}\right)$ such that $u_{k} \rightarrow u$ weakly in the $B V$-sense, $G_{u_{k}}(\omega) \rightarrow T_{u}(\omega)$ for every $\omega \in \mathcal{D}^{n, 1}\left(B^{n} \times \mathcal{Y}\right)$ and

$$
\lim _{k \rightarrow \infty} \int_{B^{n}}\left|D u_{k}\right| d x=\mathcal{E}_{T V}\left(u, B^{n}\right) .
$$

However, we recall that if $n \geq 2$ in general the current $T_{u}$ has non zero boundary in $B^{n} \times \mathcal{Y}$, even if $u \in W^{1,1}\left(B^{n}, \mathcal{Y}\right)$. 


\section{Cartesian Currents in Dimension one}

In this section we discuss some features of 1-dimensional Cartesian currents in $B^{1} \times \mathcal{Y}$ and, in particular, we discuss a gap phenomenon and the relaxed area functional. If $n=1$ we set

$$
\operatorname{cart}\left(B^{1} \times \mathcal{Y}\right):=\left\{T \in \operatorname{cart}\left(B^{1} \times \mathbb{R}^{N}\right) \mid \operatorname{spt} T \subset \bar{B}^{1} \times \mathcal{Y}\right\},
$$

where $\operatorname{cart}\left(B^{1} \times \mathbb{R}^{N}\right)$ denotes the class of integer multiplicity (say i.m.) rectifiable currents $T$ in $B^{1} \times \mathbb{R}^{N}$ such that $\partial T\left\llcorner B^{1} \times \mathbb{R}^{N}=0\right.$,

$$
\sup \left\{T(\varphi(x, y)|y| d x) \mid \varphi \in C_{c}^{0}\left(B^{1} \times \mathbb{R}^{N}\right) \text { and }\|\varphi\| \leq 1\right\}<\infty,
$$

$T(\varphi(x, y) d x) \geq 0$ if $\varphi \geq 0$ and $\pi_{\#}(T)=\llbracket B^{1} \rrbracket$, where $\pi: \mathbb{R}^{n+N} \rightarrow \mathbb{R}^{n}$ and $\widehat{\pi}: \mathbb{R}^{n+N} \rightarrow \mathbb{R}^{N}$ are the orthogonal projections onto the first $n$ and the last $N$ coordinates, respectively.

From the properties of the class of Cartesian currents $\operatorname{cart}\left(B^{n} \times \mathbb{R}^{N}\right)$, compare $\left[9\right.$, Vol. I], we infer that the class $\operatorname{cart}\left(B^{1} \times \mathcal{Y}\right)$ contains the weak limits of sequences of graphs of smooth maps $u_{k}: B^{1} \rightarrow \mathcal{Y}$ with equibounded $W^{1,1}$-energies; it is also closed under the weak convergence in $\mathcal{D}_{1}\left(B^{1} \times \mathcal{Y}\right)$ with equibounded masses. Moreover, every $T \in \operatorname{cart}\left(B^{1} \times \mathcal{Y}\right)$ is an i.m. rectifiable current in $\mathcal{R}_{1}\left(B^{1} \times \mathcal{Y}\right)$, see [6], and to $T$ it corresponds a $B V$-function $u_{T} \in B V\left(B^{1}, \mathcal{Y}\right)$ such that

$$
T(\phi(x, y) d x)=\int_{B^{n}} \phi\left(x, u_{T}(x)\right) d x
$$

for all $\phi \in C^{0}\left(B^{n} \times \mathbb{R}^{N}\right)$ such that $|\phi(x, y)| \leq C(1+|y|)$, and

$$
(-1)^{n-i} T\left(\varphi(x) \widehat{d x^{i}} \wedge d y^{j}\right)=\left\langle D_{i} u_{T}^{j}, \varphi\right\rangle:=-\int_{B^{n}} u_{T}^{j}(x) \cdot D_{i} \varphi(x) d x
$$

for all $\varphi \in C_{c}^{1}\left(B^{n}\right)$.

Canonical Decomposition. Let $T \in \operatorname{cart}\left(B^{1} \times \mathcal{Y}\right)$. Since $T$ has finite mass, $\eta \mapsto T\left(\chi_{B_{r}(x)} \wedge \eta\right)$, where $x \in B^{1}$ and $0<r<1-|x|$, defines a current in $\mathcal{D}_{1}(\mathcal{Y})$. The 1-dimensional restriction of $T$ over the point $x$

$$
\widehat{\pi}_{\#}\left(T\llcorner\{x\} \times \mathcal{Y}) \in \mathcal{D}_{1}(\mathcal{Y})\right.
$$

is the limit

$$
\widehat{\pi}_{\#}\left(T\llcorner\{x\} \times \mathcal{Y})(\eta):=\lim _{r \rightarrow 0^{+}} T\left(\chi_{B_{r}(x)} \wedge \eta\right), \quad \eta \in \mathcal{D}^{1}(\mathcal{Y}) .\right.
$$

There is a canonical way to decompose a current $T \in \operatorname{cart}\left(B^{1} \times \mathcal{Y}\right)$. We first observe that the 1-dimensional restriction of $T$ over any point $x$ in the jump set $J_{u_{T}}$ of $u_{T}$ is given by

$$
\widehat{\pi}_{\#}\left(T\llcorner\{x\} \times \mathcal{Y})=\delta_{x} \times \Gamma_{x},\right.
$$


$\Gamma_{x}$ being a 1-dimensional integral chain on $\mathcal{Y}$ such that $\partial \Gamma_{x}=\delta_{u_{T}^{+}(x)}-\delta_{u_{T}^{-}(x)}$, where $u_{T}^{+}(x)$ and $u_{T}^{-}(x)$ here and in the sequel denote the right and left limits of $u_{T}$ at $x$, respectively. Therefore, by applying Federer's decomposition theorem [6], we find an indecomposable 1-dimensional integral chain $\gamma_{x}$ on $\mathcal{Y}$, satisfying $\partial \gamma_{x}=\delta_{u_{T}^{+}(x)}-\delta_{u_{T}^{-}(x)}$, and an integral 1-cycle $C_{x}$ in $\mathcal{Y}$, satisfying $\partial C_{x}=0$, such that

$$
\Gamma_{x}=\gamma_{x}+C_{x} \quad \text { and } \quad \mathbf{M}\left(\Gamma_{x}\right)=\mathbf{M}\left(\gamma_{x}\right)+\mathbf{M}\left(C_{x}\right) .
$$

Currents associated to Graphs of $B V$-FunCtions. Next we associate to any $T \in \operatorname{cart}\left(B^{1} \times \mathcal{Y}\right)$ a current $G_{T} \in \mathcal{D}_{1}\left(B^{1} \times \mathcal{Y}\right)$ carried by the graph of the function $u_{T} \in B V\left(B^{1}, \mathcal{Y}\right)$ corresponding to $T$, and acting in a linear way on forms $\omega$ in $\mathcal{D}^{1}\left(B^{1} \times \mathcal{Y}\right)$ as follows. We first split $\omega=\omega^{(0)}+\omega^{(1)}$ according to the number of vertical differentials, see (1.5), so that

$$
\omega^{(0)}=\phi(x, y) d x \quad \text { and } \quad \omega^{(1)}=\sum_{j=1}^{N} \phi^{j}(x, y) d y^{j}, \quad \phi, \phi^{j} \in C_{0}^{\infty}\left(B^{1} \times \mathcal{Y}\right) .
$$

We then decompose $G_{T}$ into its absolutely continuous, Cantor, and Jump parts

$$
G_{T}:=T^{a}+T^{C}+T^{J}
$$

and define $T^{a}\left(\omega^{(0)}\right):=\int_{B^{1}} \phi\left(x, u_{T}(x)\right) d x, T^{C}\left(\omega^{(0)}\right)=T^{J}\left(\omega^{(0)}\right)=0$ and

$$
\begin{aligned}
T^{a}\left(\omega^{(1)}\right) & :=\sum_{j=1}^{N} \int_{B^{1}} \phi^{j}\left(x, u_{T}(x)\right) \nabla u_{T}^{j}(x) d x \\
T^{C}\left(\omega^{(1)}\right) & :=\sum_{j=1}^{N}\left\langle D^{C} u_{T}^{j}, \phi^{j}\left(\cdot, u_{T}(\cdot)\right)\right\rangle \\
T^{J}\left(\omega^{(1)}\right) & :=\sum_{j=1}^{N} \int_{J_{u_{T}}}\left(\int_{\gamma_{x}} \phi^{j}(x, y) d y^{j}\right) \cdot \nu(x) d \mathcal{H}^{0}(x),
\end{aligned}
$$

where $\gamma_{x}$ is the indecomposable 1-dimensional integral chain defined by means of the 1-dimensional restriction of $T$ over the point $x \in J_{u_{T}}$, see (2.2).

Notice that the definition of $G_{T}$ obviously depends on $\gamma_{x}$ and hence, in conclusion, on the current $T \in \operatorname{cart}\left(B^{1} \times \mathcal{Y}\right)$. Moreover, we readily infer that the mass of $G_{T}$ is given by

$$
\mathbf{M}\left(G_{T}\right)=\mathbf{M}\left(T^{a}\right)+\mathbf{M}\left(T^{C}\right)+\mathbf{M}\left(T^{J}\right),
$$

where

$\int_{B^{1}} \sqrt{1+\left|\nabla u_{T}(x)\right|^{2}} d x, \quad \mathbf{M}\left(T^{C}\right)=\left|D^{C} u_{T}\right|\left(B^{1}\right), \quad \mathbf{M}\left(T^{J}\right)=\int_{J_{u_{T}}} \mathcal{H}^{1}\left(\gamma_{x}\right) d \mathcal{H}^{0}(x)$ 
A DENSITY RESULT. If $u: B^{1} \rightarrow \mathcal{Y}$ is smooth, or at least e.g. $u \in W^{1,1}\left(B^{1}, \mathcal{Y}\right)$, and $G_{u}$ is the current carried by the graph of $u$, see (1.8), the mass of $G_{u}$ agrees with the area $\mathcal{A}(u)$ of the graph of $u$

$$
\mathbf{M}\left(G_{u}\right)=\mathcal{A}(u):=\int_{B^{1}} \sqrt{1+|D u(x)|^{2}} d x .
$$

The following strong density result for the mass of $G_{T}$ holds true.

Proposition 2.1. For every $T \in \operatorname{cart}\left(B^{1} \times \mathcal{Y}\right)$ there exists a sequence of smooth maps $\left\{u_{k}\right\} \subset C^{1}\left(B^{1}, \mathcal{Y}\right)$ such that $u_{k} \rightarrow u_{T}$ weakly in the $B V$-sense, $G_{u_{k}} \rightarrow G_{T}$ weakly in $\mathcal{D}_{1}\left(B^{1} \times \mathcal{Y}\right)$ and $\mathbf{M}\left(G_{u_{k}}\right) \rightarrow \mathbf{M}\left(G_{T}\right)$ as $k \rightarrow \infty$.

Vertical Homology. Let now $\mathcal{Z}^{1,1}\left(B^{1} \times \mathcal{Y}\right)$ denote the class of vertically closed forms

$$
\mathcal{Z}^{1,1}\left(B^{1} \times \mathcal{Y}\right):=\left\{\omega \in \mathcal{D}^{1}\left(B^{1} \times \mathcal{Y}\right) \mid d_{y} \omega^{(1)}=0\right\},
$$

where $d=d_{x}+d_{y}$ denotes the splitting of the exterior differential $d$ into a horizontal and a vertical differential. Moreover, we say that $T_{k} \rightarrow T$ weakly in $\mathcal{Z}_{1,1}\left(B^{1} \times \mathcal{Y}\right)$ if $T_{k}(\omega) \rightarrow T(\omega)$ for every $\omega \in \mathcal{Z}^{1,1}\left(B^{1} \times \mathcal{Y}\right)$.

By Proposition 2.1, since by Stokes theorem $\partial G_{u_{k}}\left\llcorner B^{1} \times \mathcal{Y}=0\right.$, whereas $G_{u_{k}} \rightarrow G_{T}$, we obtain that

$$
\partial G_{T}\left\llcorner B^{1} \times \mathcal{Y}=0 .\right.
$$

We recall that in higher dimension $n \geq 2$ in general $G_{T}$ has interior boundary, i.e., $\partial G_{T}\left\llcorner B^{n} \times \mathcal{Y} \neq 0\right.$. Setting then

$$
S_{T}:=T-G_{T},
$$

by (2.1) we infer that $S_{T}(\phi(x, y) d x)=0$ and $S_{T}(d \phi)=0$ for every $\phi \in C_{0}^{\infty}\left(B^{1} \times\right.$ $\mathcal{Y})$. Therefore, by homological reasons, since

$$
\inf \left\{\mathbf{M}(C) \mid C \in \mathcal{Z}_{1}(\mathcal{Y}), C \text { is non trivial in } \mathcal{Y}\right\}>0,
$$

similarly to [9, Vol. II, Sec. 5.3.1] we infer that

$$
S_{T}=\sum_{i=1}^{I} \delta_{x_{i}} \times C_{i} \quad \text { on } \quad \mathcal{Z}^{1,1}\left(B^{1} \times \mathcal{Y}\right),
$$

where $\left\{x_{i}: i=1, \ldots, I\right\}$ is a finite disjoint set of points in $B^{1}$, possibly intersecting the Jump set $J_{u_{T}}$, and $C_{i}$ is a non-trivial homological integral 1-cycle in $\mathcal{Y}$. Note that the integral 1-homology group $H_{1}(\mathcal{Y})$ is finitely generated, being torsion-free.

Remark 2.2. Setting

$$
S_{T, s i n g}:=T-G_{T}-\sum_{i=1}^{I} \delta_{x_{i}} \times C_{i},
$$


it turns out that $S_{T, s i n g}$ is nonzero only possibly on forms $\omega$ with non-zero vertical component, $\omega^{(1)} \neq 0$, and such that $d_{y} \omega^{(1)} \neq 0$. Therefore, $S_{T, s i n g}$ is a homologically trivial i.m. rectifiable current in $\mathcal{R}_{1}\left(B^{1} \times \mathcal{Y}\right)$.

As a consequence, setting

$$
T^{H}:=\sum_{i=1}^{I} \delta_{x_{i}} \times C_{i},
$$

every $T \in \operatorname{cart}\left(B^{1} \times \mathcal{Y}\right)$ decomposes into its absolutely continuous, Cantor, Jump, Homological, and Singular parts,

$$
T=T^{a}+T^{C}+T^{J}+T^{H}+S_{T, s i n g} .
$$

Gap Phenomenon. However, a gap phenomenon occurs in $\operatorname{cart}\left(B^{1} \times \mathcal{Y}\right)$. More precisely, if we set

$\widetilde{\mathbf{M}}(T):=\inf \left\{\liminf _{k \rightarrow \infty} \mathbf{M}\left(G_{u_{k}}\right) \mid\left\{u_{k}\right\} \subset C^{1}\left(B^{1}, \mathcal{Y}\right), G_{u_{k}} \rightarrow T\right.$ weakly in $\left.\mathcal{D}_{1}\left(B^{1} \times \mathcal{Y}\right)\right\}$, we see that there exist Cartesian currents $T \in \operatorname{cart}\left(B^{1} \times \mathcal{Y}\right)$ for which

$$
\mathbf{M}(T)<\widetilde{\mathbf{M}}(T) .
$$

Example 2.3. Following [9, Vol. I, Sec. 4.2.5], if $T=G_{u}+\delta_{0} \times C$, where $u \equiv P \in \mathcal{Y}$ is a constant map and $C \in \mathcal{Z}^{1}(\mathcal{Y})$ is a 1-cycle in $\mathcal{Y}$, it readily follows that for every smooth sequence $\left\{u_{k}\right\} \subset C^{1}\left(B^{1}, \mathcal{Y}\right)$ such that $G_{u_{k}} \rightarrow T$ weakly in $\mathcal{D}_{1}\left(B^{1} \times \mathcal{Y}\right)$ we have that

$$
\liminf _{k \rightarrow \infty} \mathbf{M}\left(G_{u_{k}}\right) \geq \mathbf{M}(T)+2 d, \quad d:=\operatorname{dist}_{\mathcal{Y}}(P, \operatorname{spt} C),
$$

where disty denotes the geodesic distance in $\mathcal{Y}$. In fact, clearly $\mathbf{M}(T)=$ $\mathcal{L}^{1}\left(B^{1}\right)+\mathbf{M}(C)$, whereas for every $\varepsilon>0$, if $k$ is sufficiently large we have that $u_{k}:(-\varepsilon, \varepsilon) \rightarrow \mathcal{Y}$ has to connect twice the point $P$ to the support of $C$ and to parameterize the 1-cycle $C$, so that

$$
\int_{-\varepsilon}^{\varepsilon}\left|D u_{k}\right| d x \geq 2 d+\mathbf{M}(C) .
$$

Remark 2.4. This gap phenomenon is due to the structure of the area integrand $u \mapsto \sqrt{1+|D u|^{2}}$, and it is typical of integrands with linear growth of the gradient, e.g., the total variation integrand $u \mapsto|D u|$. This does not happen e.g. for the Dirichlet integrand $u \mapsto \frac{1}{2}|D u|^{2}$ in dimension 2, compare [10]. In this case, in fact, the connection from one point $P$ to any 2-cycle $C \in \mathcal{Z}^{2}(\mathcal{Y})$ can be obtained by means of "cylinders" of small 2-dimensional mapping area and, therefore, of small Dirichlet integral, on account of Morrey's $\varepsilon$-conformality theorem.

HOMOLOGICAL THEORY. In order to study the currents which arise as weak limits of graphs of smooth maps $u_{k}: B^{1} \rightarrow \mathcal{Y}$ with equibounded total variation, 
$\sup _{k}\left\|D u_{k}\right\|_{L^{1}}<\infty$, the previous facts suggest us to consider vertical homology equivalence classes of currents in $\operatorname{cart}\left(B^{1} \times \mathcal{Y}\right)$. More precisely, we give the following

Definition 2.5. We denote by $\operatorname{cart}^{1,1}\left(B^{1} \times \mathcal{Y}\right)$ the set of equivalence classes of currents in $\operatorname{cart}\left(B^{1} \times \mathcal{Y}\right)$, where

$$
T \sim \widetilde{T} \Longleftrightarrow T(\omega)=\widetilde{T}(\omega) \quad \forall \omega \in \mathcal{Z}^{1,1}\left(B^{1} \times \mathcal{Y}\right) .
$$

We observe that if $T \sim \widetilde{T}$, then the underlying $B V$-functions coincide, i.e., $u_{T}=u_{\widetilde{T}}$. Therefore, we have $T^{a}=\widetilde{T}^{a}$ and $T^{C}=\widetilde{T}^{C}$, whereas in general $T^{J} \neq \widetilde{T}^{J}$. However, we have that

$$
T^{J}+T^{H}=\widetilde{T}^{J}+\widetilde{T}^{H} \quad \text { on } \quad \mathcal{Z}^{1,1}\left(B^{1} \times \mathcal{Y}\right) .
$$

Jump-CONCENTRATION POINTS. For future use, we let

$$
J_{c}(T):=J_{u_{T}} \cup\left\{x_{i}: i=1, \ldots, I\right\}
$$

denote the set of points of jump and concentration, where the $x_{i}$ 's are given by (2.3). We infer that $J_{c}(T)$ is an at most countable set which does not depend on the representative $T$, i.e., $J_{c}(T)=J_{c}(\widetilde{T})$ if $T \sim \widetilde{T}$. By extending the notion of 1-dimensional restriction $\widehat{\pi}_{\#}(T\llcorner\{x\} \times \mathcal{Y})$ to equivalence classes, we infer that $\widehat{\pi}_{\#}\left(T\llcorner\{x\} \times \mathcal{Y})=0\right.$ if $x \notin J_{c}(T)$. As to jump-concentration points, letting

$$
\mathcal{Z}^{1}(\mathcal{Y}):=\left\{\eta \in \mathcal{D}^{1}(\mathcal{Y}) \mid d_{y} \eta=0\right\}
$$

if $x \in J_{u_{T}}$, with $x \neq x_{i}$, we infer that

$$
\widehat{\pi}_{\#}\left(T\llcorner\{x\} \times \mathcal{Y})=\gamma_{x} \quad \text { on } \quad \mathcal{Z}^{1}(\mathcal{Y}),\right.
$$

where $\gamma_{x}$ is the indecomposable 1-dimensional integral chain previously defined, and if $x=x_{i}$, see (2.4),

$$
\widehat{\pi}_{\#}\left(T\llcorner\{x\} \times \mathcal{Y})=\gamma_{x_{i}}+C_{i} \quad \text { on } \quad \mathcal{Z}^{1}(\mathcal{Y}),\right.
$$

where $C_{i} \in \mathcal{Z}_{1}(\mathcal{Y})$ is the non-trivial 1-cycle defined by (2.3), and $\gamma_{x_{i}}=0$ if $x_{i} \notin J_{u_{T}}$.

VerticAl Minimal CONNECTION. For every Cartesian current $T \in \operatorname{cart}^{1,1}\left(B^{1} \times\right.$ $\mathcal{Y})$ and every point $x \in J_{c}(T)$ we will denote by

$$
\begin{aligned}
& \Gamma_{T}(x):=\{\gamma \in \operatorname{Lip}([0,1], \mathcal{Y}) \mid \gamma(0)=u_{T}^{-}(x), \quad \gamma(1)=u_{T}^{+}(x), \\
& \gamma_{\#} \llbracket(0,1) \rrbracket(\eta)=\widehat{\pi}_{\#}\left(T\llcorner\{x\} \times \mathcal{Y})(\eta) \quad \forall \eta \in \mathcal{Z}^{1}(\mathcal{Y})\right\}
\end{aligned}
$$

the family of all smooth curves $\gamma$ in $\mathcal{Y}$, with end points $u_{T}^{ \pm}(x)$, such that their image current $\gamma_{\#} \llbracket(0,1) \rrbracket$ agrees with the 1-dimensional restriction $\widehat{\pi}_{\#}(T\llcorner\{x\} \times$ $\mathcal{Y}$ ) on closed 1 -forms in $\mathcal{Z}^{1}(\mathcal{Y})$. Moreover, we denote by

$$
\mathcal{L}_{T}(x):=\inf \left\{\mathcal{L}(\gamma) \mid \gamma \in \Gamma_{T}(x)\right\}, \quad x \in J_{c}(T),
$$


the minimal length of curves $\gamma$ connecting the "vertical part" of $T$ over $x$ to the graph of $u_{T}$. We remark that the infimum in (2.6) is attained, i.e.,

$$
\forall x \in J_{c}(T), \quad \exists \gamma \in \Gamma_{T}(x) \quad: \quad \mathcal{L}(\gamma)=\mathcal{L}_{T}(x) .
$$

RELAXED AREA FUNCTIONAL. Let us now introduce the functional

$$
\mathcal{A}(T):=\int_{B^{1}} \sqrt{1+\left|\nabla u_{T}(x)\right|^{2}} d x+\left|D^{C} u_{T}\right|\left(B^{1}\right)+\int_{J_{c}(T)} \mathcal{L}_{T}(x) d \mathcal{H}^{0}(x) .
$$

Note that for every $T \in \operatorname{cart}^{1,1}\left(B^{1} \times \mathcal{Y}\right)$ we have

$$
\min \{\mathbf{M}(\widetilde{T}): \widetilde{T} \sim T\} \leq \mathcal{A}(T) .
$$

The following lower semicontinuity property holds.

Theorem 2.6. Let $T \in \operatorname{cart}^{1,1}\left(B^{1} \times \mathcal{Y}\right)$. For every sequence of smooth maps $\left\{u_{k}\right\} \subset C^{1}\left(B^{1}, \mathcal{Y}\right)$ such that $G_{u_{k}} \rightarrow T$ weakly in $\mathcal{Z}_{1,1}\left(B^{1} \times \mathcal{Y}\right)$, we have

$$
\liminf _{k \rightarrow \infty} \mathbf{M}\left(G_{u_{k}}\right) \geq \mathcal{A}(T) \text {. }
$$

Moreover, we also have the following density result.

Theorem 2.7. Let $T \in \operatorname{cart}^{1,1}\left(B^{1} \times \mathcal{Y}\right)$. There exists a sequence of smooth maps $\left\{u_{k}\right\} \subset C^{1}\left(B^{1}, \mathcal{Y}\right)$ such that $G_{u_{k}} \rightarrow T$ weakly in $\mathcal{Z}_{1,1}\left(B^{1} \times \mathcal{Y}\right)$ and $\mathbf{M}\left(G_{u_{k}}\right) \rightarrow \mathcal{A}(T)$ as $k \rightarrow \infty$.

As a consequence, if we denote, in the same spirit as Lebesgue's relaxed area, $\widetilde{\mathcal{A}}(T):=\inf \left\{\liminf _{k \rightarrow \infty} \mathcal{A}\left(u_{k}\right) \mid\left\{u_{k}\right\} \subset C^{1}\left(B^{1}, \mathcal{Y}\right), G_{u_{k}} \rightarrow T\right.$ weakly in $\left.\mathcal{Z}_{1,1}\left(B^{1} \times \mathcal{Y}\right)\right\}$, we conclude by Theorems 2.6 and 2.7 that

$$
\mathcal{A}(T)=\widetilde{\mathcal{A}}(T) \quad \forall T \in \operatorname{cart}^{1,1}\left(B^{1} \times \mathcal{Y}\right) .
$$

From Theorems 2.6 and 2.7, (2.7) and the closure of the class $\operatorname{cart}\left(B^{1} \times \mathcal{Y}\right)$ we infer:

(i) the functional $T \mapsto \mathcal{A}(T)$ is lower semicontinuous in $\operatorname{cart}^{1,1}\left(B^{1} \times \mathcal{Y}\right)$ w.r.t. the weak convergence in $\mathcal{Z}_{1,1}\left(B^{1} \times \mathcal{Y}\right)$;

(ii) the class $\operatorname{cart}^{1,1}\left(B^{1} \times \mathcal{Y}\right)$ is closed and compact under weak convergence in $\mathcal{Z}_{1,1}\left(B^{1} \times \mathcal{Y}\right)$ with equibounded $\mathcal{A}$-energies.

We finally notice that similar properties hold if one considers the total variation integrand $u \mapsto|D u|$ instead of the area integrand $u \mapsto \sqrt{1+|D u|^{2}}$. In particular, setting

$$
\mathcal{E}_{1,1}(T):=\int_{B^{1}}\left|\nabla u_{T}(x)\right| d x+\left|D^{C} u_{T}\right|\left(B^{1}\right)+\int_{J_{c}(T)} \mathcal{L}_{T}(x) d \mathcal{H}^{0}(x),
$$


compare Sec. 3 below, for every $T \in \operatorname{cart}^{1,1}\left(B^{1} \times \mathcal{Y}\right)$ we have $\mathcal{E}_{1,1}(T)=\inf \{$ $\liminf _{k \rightarrow \infty} \int_{B^{1}}\left|D u_{k}\right| d x \mid\left\{u_{k}\right\} \subset C^{1}\left(B^{1}, \mathcal{Y}\right), \quad G_{u_{k}} \rightarrow T \quad$ weakly in $\mathcal{Z}_{1,1}\left(B^{1} \times\right.$ $\mathcal{Y})\}$.

\section{Cartesian Currents, BV-energy and weak limits}

In this section we deal with the weak limits of graphs of smooth maps $u_{k}$ : $B^{n} \rightarrow \mathcal{Y}$, in any dimension $n$, with equibounded $W^{1,1}$-energies. We first state a few preliminary results.

Homological FaCTs. Since $H_{1}(\mathcal{Y})$ has no torsion, there are generators $\left[\gamma_{1}\right], \ldots,\left[\gamma_{\bar{s}}\right]$, i.e. integral 1-cycles in $\mathcal{Z}_{1}(\mathcal{Y})$, such that

$$
H_{1}(\mathcal{Y})=\left\{\sum_{s=1}^{\bar{s}} n_{s}\left[\gamma_{s}\right] \mid n_{s} \in \mathbb{Z}\right\} .
$$

By de Rham's theorem the first real homology group is in duality with the first cohomology group $H_{d R}^{1}(\mathcal{Y})$, the duality being given by the natural pairing

$$
\langle[\gamma],[\omega]\rangle:=\gamma(\omega)=\int_{\gamma} \omega, \quad[\gamma] \in H_{1}(\mathcal{Y} ; \mathbb{R}), \quad[\omega] \in H_{d R}^{1}(\mathcal{Y})
$$

We will then denote by $\left[\omega^{1}\right], \ldots,\left[\omega^{\bar{s}}\right]$ a dual basis in $H_{d R}^{1}(\mathcal{Y})$ so that $\gamma_{s}\left(\omega^{r}\right)=$ $\delta_{s r}$, where $\delta_{s r}$ denotes the Kronecker symbols.

$\mathcal{D}_{p, 1}$-CURRENTS AND BOUNDARIES. For $p=1, \ldots, n$, we denote by $\mathcal{D}^{p, 1}\left(B^{n} \times\right.$ $\mathcal{Y})$ the subspace of compactly supported smooth $p$-forms in $\mathcal{D}^{p}\left(B^{n} \times \mathcal{Y}\right)$ of the type $\omega=\omega^{(0)}+\omega^{(1)}$, where $\omega^{(j)}$ denotes the component that contain exactly $j$ differentials in the vertical $\mathcal{Y}$ variables. We also denote by $\mathcal{D}_{p, 1}\left(B^{n} \times \mathcal{Y}\right)$ the dual space of $\mathcal{D}^{p, 1}\left(B^{n} \times \mathcal{Y}\right)$. For example, if $u \in W^{1,1}\left(B^{n}, \mathcal{Y}\right)$, then $G_{u}$ is an $(n, 1)$-current in $\mathcal{D}_{n, 1}\left(B^{n} \times \mathcal{Y}\right)$ defined in an approximate sense by $(1.8)$.

If $\left\{T_{k}\right\} \subset \mathcal{D}_{p, 1}\left(B^{n} \times \mathcal{Y}\right)$, we say that $\left\{T_{k}\right\}$ converges weakly in $\mathcal{D}_{p, 1}\left(B^{n} \times \mathcal{Y}\right)$, $T_{k} \rightarrow T$, if $T_{k}(\omega) \rightarrow T(\omega)$ for every $\omega \in \mathcal{D}^{p, 1}\left(B^{n} \times \mathcal{Y}\right)$. Trivially, the class $\mathcal{D}_{p, 1}\left(B^{n} \times \mathcal{Y}\right)$ is closed under weak convergence.

The exterior differential $d$ splits into a horizontal and a vertical differential $d=d_{x}+d_{y}$. Of course $\partial_{x} T(\omega):=T\left(d_{x} \omega\right)$ defines a boundary operator $\partial_{x}$ : $\mathcal{D}_{n, 1}\left(B^{n} \times \mathcal{Y}\right) \rightarrow \mathcal{D}_{n-1,1}\left(B^{n} \times \mathcal{Y}\right)$. Now, for any $\omega \in \mathcal{D}^{n-1,1}\left(B^{n} \times \mathcal{Y}\right), d_{y} \omega$ belongs to $\mathcal{D}^{n, 1}\left(B^{n} \times \mathcal{Y}\right)$ if and only if $d_{y} \omega^{(1)}=0$. Then $\partial_{y} T$ makes sense only as an element of the dual space of $\mathcal{Z}^{n-1,1}\left(B^{n} \times \mathcal{Y}\right)$, where

$$
\mathcal{Z}^{p, 1}\left(B^{n} \times \mathcal{Y}\right):=\left\{\omega \in \mathcal{D}^{p, 1}\left(B^{n} \times \mathcal{Y}\right) \mid d_{y} \omega^{(1)}=0\right\} .
$$


$\mathcal{E}_{1,1}$-NORM. For any $\omega \in \mathcal{D}^{n, 1}\left(B^{n} \times \mathcal{Y}\right)$ and $T \in \mathcal{D}_{n, 1}\left(B^{n} \times \mathcal{Y}\right)$ we set

$$
\begin{aligned}
& \|\omega\|_{\mathcal{E}_{1,1}}:=\max \left\{\sup _{x, y} \frac{\left|\omega^{(0)}(x, y)\right|}{1+|y|}, \int_{B^{n}} \sup _{y}\left|\omega^{(1)}(x, y)\right| d x\right\}, \\
& \|T\|_{\mathcal{E}_{1,1}}:=\sup \left\{T(\omega) \mid \omega \in \mathcal{D}^{n, 1}\left(B^{n} \times \mathcal{Y}\right),\|\omega\|_{\mathcal{E}_{1,1} \leq 1} \leq .\right.
\end{aligned}
$$

It is not difficult to show that $\|T\|_{\mathcal{E}_{1,1}}$ is a norm on $\left\{T \in \mathcal{D}_{n, 1}\left(B^{n} \times \mathcal{Y}\right)\right.$ : $\left.\|T\|_{\mathcal{E}_{1,1}}<\infty\right\}$. Moreover, $\|\cdot\|_{\mathcal{E}_{1,1}}$ is weakly lower semicontinuous in $\mathcal{D}_{n, 1}$, so that $\left\{T \in \mathcal{D}_{n, 1}\left(B^{n} \times \mathcal{Y}\right):\|T\|_{\mathcal{E}_{1,1}}<\infty\right\}$ is closed under weak $\mathcal{D}_{n, 1}$-convergence with equibounded $\mathcal{E}_{1,1}$-norms. Finally, if $\sup _{k}\left\|T_{k}\right\|_{\mathcal{E}_{1,1}}<\infty$ there is a subsequence which weakly converges to some $T \in \mathcal{D}_{n, 1}\left(B^{n} \times \mathcal{Y}\right)$ with $\|T\|_{\mathcal{E}_{1,1}}<\infty$.

WEAK LIMITS OF SMOOTH GRAPHS. Let $\left\{u_{k}\right\} \subset C^{1}\left(B^{n}, \mathcal{Y}\right)$ be a sequence of smooth maps with equibounded $W^{1,1}$-energies, $\sup _{k}\left\|D u_{k}\right\|_{L^{1}}<\infty$. The currents $G_{u_{k}}$ carried by the graphs of the $u_{k}$ 's are well defined currents in $\mathcal{D}_{n, 1}\left(B^{n} \times\right.$ $\mathcal{Y})$ with equibounded $\mathcal{E}_{1,1}$-norms. Possibly passing to a subsequence, we then infer that $G_{u_{k}} \rightarrow T$ weakly in $\mathcal{D}_{n, 1}\left(B^{n} \times \mathcal{Y}\right)$ to some current $T \in \mathcal{D}_{n, 1}\left(B^{n} \times \mathcal{Y}\right)$, and $u_{k} \rightarrow u_{T}$ weakly in the $B V$-sense to some function $u_{T} \in B V\left(B^{n}, \mathcal{Y}\right)$. Therefore, we clearly have that

$$
T(\phi(x, y) d x)=\int_{B^{n}} \phi\left(x, u_{T}(x)\right) d x \quad \forall \phi \in C_{c}^{\infty}\left(B^{n} \times \mathcal{Y}\right) .
$$

Moreover, by lower semicontinuity we have $\|T\|_{\mathcal{E}_{1,1}}<\infty$ whereas, since the $G_{u_{k}}$ 's have no boundary in $B^{n} \times \mathcal{Y}$, by the weak convergence we also infer

$$
\partial T=0 \quad \text { on } \mathcal{Z}^{n-1,1}\left(B^{n} \times \mathcal{Y}\right) .
$$

CURRENTS ASSOCIATED TO GRAPHS OF $B V$-FUnCTIONS. As in the previous section, we associate to the weak limit current $T \in \mathcal{D}_{n, 1}\left(B^{n} \times \mathcal{Y}\right)$ a current $G_{T} \in \mathcal{D}_{n, 1}\left(B^{n} \times \mathcal{Y}\right)$ carried by the graph of the corresponding function $u_{T} \in$ $B V\left(B^{n}, \mathcal{Y}\right)$, acting on forms $\omega$ in $D^{n, 1}\left(B^{n} \times \mathcal{Y}\right)$ as follows. We decompose $G_{T}$ into its absolutely continuous, Cantor, and Jump parts

$$
G_{T}:=T^{a}+T^{C}+T^{J} .
$$

If $\omega=\omega^{(0)}+\omega^{(1)}$, where $\omega^{(0)}$ and $\omega^{(1)}$ are given by (1.5), we define $G_{T}\left(\omega^{(0)}\right)=$ $T^{a}\left(\omega^{(0)}\right)$, where $T^{a}\left(\omega^{(0)}\right)$ is given by (1.6), where this time $u=u_{T}$, and we set $T^{C}\left(\omega^{(0)}\right)=T^{J}\left(\omega^{(0)}\right)=0$. Moreover, we define $T^{a}\left(\omega^{(1)}\right), T^{C}\left(\omega^{(1)}\right)$, and $T^{J}\left(\omega^{(1)}\right)$ as in (1.7), with $T$ instead of $T_{u}$, where $\gamma_{x}$ is the indecomposable 1-dimensional integral chain defined as in the previous section. We will see that $\gamma_{x}$ is well-defined for $\mathcal{H}^{n-1}$-a.e. $x \in J_{u_{T}}$, since $\|T\|_{\mathcal{E}_{1,1}}<\infty$, compare (2.2) and Definition 3.5 below. In general $\partial G_{T}\left\llcorner B^{n} \times \mathcal{Y} \neq 0\right.$. However, setting

$$
S_{T}:=T-G_{T},
$$


we clearly have $S_{T}(\phi(x, y) d x)=0$ for every $\phi \in C_{c}^{\infty}\left(B^{n} \times \mathcal{Y}\right)$. Moreover, we also have:

Proposition 3.1. $S_{T}(\omega)=0$ for every form $\omega=\omega^{(1)}$ such that $\omega=d_{y} \widetilde{\omega}$ for some $\widetilde{\omega} \in \mathcal{D}^{n-1,0}\left(B^{n} \times \mathcal{Y}\right)$.

Proof. Write $\widetilde{\omega}:=\omega_{\varphi} \wedge \eta$ for some $\eta \in C_{0}^{\infty}(\mathcal{Y})$ and $\varphi=\left(\varphi^{1}, \ldots, \varphi^{n}\right) \in$ $C_{0}^{\infty}\left(B^{n}, \mathbb{R}^{n}\right)$, where

$$
\omega_{\varphi}:=\sum_{i=1}^{n}(-1)^{i-1} \varphi^{i}(x) \widehat{d x^{i}}
$$

Since

and $T\left(d\left(\omega_{\varphi} \wedge \eta\right)\right)=\partial T\left(\omega_{\varphi} \wedge \eta\right)=0$, we have

$$
d\left(\omega_{\varphi} \wedge \eta\right)=\operatorname{div} \varphi(x) \eta(y) d x+(-1)^{n-1} \omega_{\varphi} \wedge d_{y} \eta
$$

$$
(-1)^{n} T(\operatorname{div} \varphi(x) \eta(y) d x)=T\left(\omega_{\varphi} \wedge d_{y} \eta\right)
$$

so that

$$
S_{T}\left(\omega_{\varphi} \wedge d_{y} \eta\right)=(-1)^{n} T(\operatorname{div} \varphi(x) \eta(y) d x)-G_{T}\left(\omega_{\varphi} \wedge d_{y} \eta\right) .
$$

Moreover, since $T_{(0)}=G_{T(0)}$, by (1.6) we have

$$
T(\operatorname{div} \varphi(x) \eta(y) d x)=\int_{B^{n}} \operatorname{div} \varphi(x) \eta\left(u_{T}(x)\right) d x=-\left\langle D\left(\eta \circ u_{T}\right), \varphi\right\rangle
$$

whereas, taking $\phi_{i}^{j}=\varphi^{i} D_{y_{j}} \eta$ in (1.5), by the definition of $G_{T}$, since $\partial \gamma_{x}=$ $\delta_{u_{T}^{+}(x)}-\delta_{u_{T}^{-}(x)}$ we infer

$$
\begin{aligned}
(-1)^{n-1} G_{T}\left(\omega_{\varphi} \wedge d_{y} \eta\right) & =\sum_{j=1}^{N} \int_{B^{n}} \frac{\partial \eta}{\partial y^{j}}\left(u_{T}(x)\right)\left\langle\nabla u_{T}^{j}(x), \varphi(x)\right\rangle d x \\
& +\sum_{j=1}^{N} \int_{B^{n}} \frac{\partial \eta}{\partial y^{j}}\left(u_{T}(x)\right) \varphi(x) d D^{C} u_{T}^{j} \\
& +\int_{J_{u_{T}}}\left(\eta\left(u_{T}^{+}(x)\right)-\eta\left(u_{T}^{-}(x)\right)\langle\varphi(x), \nu(x)\rangle d \mathcal{H}^{n-1}\right.
\end{aligned}
$$

Finally, by the chain rule for the derivative $D\left(\eta \circ u_{T}\right)$ we obtain

$$
(-1)^{n-1} G_{T}\left(\omega_{\varphi} \wedge d_{y} \eta\right)=\left\langle D\left(\eta \circ u_{T}\right), \varphi\right\rangle
$$

and hence that $S_{T}\left(\omega_{\varphi} \wedge d_{y} \eta\right)=0$.

In conclusion, similarly to [9], Vol. II, Sec. 5.4.3, we infer that the weak limit current $T$ is given by

$$
T=G_{T}+S_{T}, \quad S_{T}=\sum_{s=1}^{\bar{s}} \mathbb{L}_{s}(T) \times \gamma_{s} \quad \text { on } \quad \mathcal{Z}^{n, 1}\left(B^{n} \times \mathcal{Y}\right),
$$


where $\mathbb{L}_{s}(T) \in \mathcal{D}_{n-1}\left(B^{n}\right)$ is defined by

$$
\mathbb{L}_{s}(T)(\phi):=S_{T}\left(\pi^{\#} \phi \wedge \widehat{\pi}^{\#} \omega^{s}\right) \quad \forall \phi \in \mathcal{D}^{n-1}\left(B^{n}\right) .
$$

Finally, setting

$$
S_{T, \text { sing }}:=T-G_{T}-\sum_{s=1}^{\bar{s}} \mathbb{L}_{s}(T) \times \gamma_{s},
$$

see Remark 2.2 , it turns out that $S_{T, s i n g}$ is nonzero only possibly on forms $\omega$ with non-zero vertical component, $\omega^{(1)} \neq 0$, and such that $d_{y} \omega^{(1)} \neq 0$.

PARAMETRIC POLYCONVEX L.S.C. EXTENSION OF THE TOTAL VARIATION. We recall from [9], Vol. II, Sec. 1.2 that the parametric variational integral associated to the total variation integral is a well-defined functional $T \mapsto \mathcal{F}_{1,1}(T)$ on currents $T \in \mathcal{D}_{n, 1}\left(B^{n} \times \mathcal{Y}\right)$ satisfying $\|T\|_{\mathcal{E}_{1,1}}<\infty$. It is lower semicontinuous with respect to the weak convergence in $\mathcal{D}_{n, 1}\left(B^{n} \times \mathcal{Y}\right)$, and satisfies

$$
\mathcal{F}_{1,1}\left(G_{u}\right)=\int_{B^{n}}|D u(x)| d x
$$

if $T=G_{u}$ for some $u \in W^{1,1}\left(B^{n}, \mathcal{Y}\right)$, see (1.8). Now, if $T \in \mathcal{D}_{n, 1}\left(B^{n} \times \mathcal{Y}\right)$ is the weak limit of a sequence $\left\{G_{u_{k}}\right\}$ of graphs of smooth maps $\left\{u_{k}\right\} \subset C^{1}\left(B^{n}, \mathcal{Y}\right)$ with equibounded $W^{1,1}$-energies, by lower semicontinuity we have $\mathcal{F}_{1,1}(T)<\infty$. Moreover, writing $T$ as in (3.3), we have

$$
\mathcal{F}_{1,1}(T)=\mathcal{F}_{1,1}\left(G_{T}\right)+\mathcal{F}_{1,1}\left(S_{T}\right)
$$

where

$$
\mathcal{F}_{1,1}\left(G_{T}\right)=\int_{B^{n}}\left|\nabla u_{T}(x)\right| d x+\left|D^{C} u_{T}\right|\left(B^{n}\right)+\int_{J_{u_{T}}} \mathcal{H}^{1}\left(\gamma_{x}\right) d \mathcal{H}^{n-1}(x) .
$$

In addition, if the singular part $S_{T, s i n g}$ defined in (3.5) vanishes, and if the $\mathbb{L}_{s}(T)$ 's are i.m. rectifiable currents in $\mathcal{R}_{n-1}\left(B^{n}\right)$, we have

$$
\mathcal{F}_{1,1}\left(S_{T}\right)=\sum_{s=1}^{\bar{s}} \mathbf{M}\left(\mathbb{L}_{s}(T)\right) \cdot \mathbf{M}\left(\gamma_{s}\right) .
$$

Gap Phenomenon. Similarly to the case of dimension $n=1$, a gap phenomenon occurs. More precisely, in general for every smooth sequence $\left\{u_{k}\right\} \subset$ $C^{1}\left(B^{n}, \mathcal{Y}\right)$ such that $G_{u_{k}} \rightarrow T$ weakly in $\mathcal{D}_{n, 1}\left(B^{n} \times \mathcal{Y}\right)$ we have that

$$
\liminf _{k \rightarrow \infty} \mathcal{F}_{1,1}\left(G_{u_{k}}\right) \geq \mathcal{F}_{1,1}(T)+C
$$

for some absolute constant $C>0$, see Remark 2.4.

Vertical HOMOLOGY CLASSES. In analogy with the previous section, Definition 2.5, we then consider vertical homology equivalence classes of currents satisfying the same structure properties as weak limits of graphs of smooth maps 
$u_{k}: B^{n} \rightarrow \mathcal{Y}$ with equibounded total variation, $\sup _{k}\left\|D u_{k}\right\|_{L^{1}}<\infty$. More precisely, we say that

$$
T \sim \widetilde{T} \Longleftrightarrow T(\omega)=\widetilde{T}(\omega) \quad \forall \omega \in \mathcal{Z}^{n, 1}\left(B^{n} \times \mathcal{Y}\right) .
$$

Moreover, we will say that $T_{k} \rightarrow T$ weakly in $\mathcal{Z}_{n, 1}\left(B^{n} \times \mathcal{Y}\right)$ if $T_{k}(\omega) \rightarrow T(\omega)$ for every $\omega \in \mathcal{Z}^{n, 1}\left(B^{n} \times \mathcal{Y}\right)$.

$B V$-GRAPHS. Extending the structure properties of the current $G_{T} \in \mathcal{D}_{n, 1}\left(B^{n} \times\right.$ $\mathcal{Y})$ carried by the graph of $u_{T} \in B V\left(B^{n}, \mathcal{Y}\right)$ and associated to the weak limit current $T \in \mathcal{D}_{n, 1}\left(B^{n} \times \mathcal{Y}\right)$, we give the following

Definition 3.2. A current $G \in \mathcal{D}_{n, 1}\left(B^{n} \times \mathcal{Y}\right)$ is said to be in $B V-\operatorname{graph}\left(B^{n} \times\right.$ $\mathcal{Y})$ if there exists a function $u=u(G) \in B V\left(B^{n}, \mathcal{Y}\right)$ and for $\mathcal{H}^{n-1}$-a.e. $\quad x \in J_{u}$ a 1-dimensional integral chain $\gamma_{x}$ in $\mathcal{Y}$, satisfying $\partial \gamma_{x}=\delta_{u^{+}(x)}-\delta_{u^{-}(x)}$, for which the following structure properties hold. $G$ decomposes into its absolutely continuous, Cantor, and Jump parts

$$
G:=G^{a}+G^{C}+G^{J} .
$$

If $\omega=\omega^{(0)}+\omega^{(1)}$, where $\omega^{(0)}$ and $\omega^{(1)}$ are given by $(1.5)$, we have $G\left(\omega^{(0)}\right):=$ $G^{a}\left(\omega^{(0)}\right)$, where $G^{a}\left(\omega^{(0)}\right)$ is given by $(1.6)$, and $G^{C}\left(\omega^{(0)}\right)=G^{J}\left(\omega^{(0)}\right)=0$. Moreover, $G^{a}\left(\omega^{(1)}\right), G^{C}\left(\omega^{(1)}\right)$, and $G^{J}\left(\omega^{(1)}\right)$ are given by (1.7), with $G$ instead of $T_{u}$, where $\gamma_{x}$ is previously given.

As already noticed, in general $\partial G\left\llcorner B^{n} \times \mathcal{Y} \neq 0\right.$.

Definition 3.3. We denote by $\mathcal{E}_{1,1}-\operatorname{graph}\left(B^{n} \times \mathcal{Y}\right)$ the set of equivalence classes, in the sense of (3.6), of currents $T$ in $\mathcal{D}_{n, 1}\left(B^{n} \times \mathcal{Y}\right)$ which have no interior boundary,

$$
\partial T=0 \quad \text { on } \quad \mathcal{Z}^{n-1,1}\left(B^{n} \times \mathcal{Y}\right),
$$

finite $\mathcal{E}_{1,1}$-norm, i.e.

$$
\|T\|_{\mathcal{E}_{1,1}}:=\sup \left\{T(\omega) \mid \omega \in \mathcal{Z}^{n, 1}\left(B^{n} \times \mathcal{Y}\right),\|\omega\|_{\mathcal{E}_{1,1}} \leq 1\right\}<\infty
$$

and decompose as

$$
T=G_{T}+S_{T}, \quad S_{T}=\sum_{s=1}^{\bar{s}} \mathbb{L}_{s}(T) \times \gamma_{s} \quad \text { on } \quad \mathcal{Z}^{n, 1}\left(B^{n} \times \mathcal{Y}\right),
$$

where $G_{T} \in B V-\operatorname{graph}\left(B^{n} \times \mathcal{Y}\right)$, see Definition 3.2 , and $\mathbb{L}_{s}(T)$ is an i.m. rectifiable current in $\mathcal{R}_{n-1}\left(B^{n}\right)$ for every s.

Remark 3.4. Note that if $\widetilde{T} \sim T$, in general $G_{\widetilde{T}} \neq G_{T}$. However, the corresponding $B V$-functions coincide, i.e., $u\left(G_{T}\right)=u\left(G_{\widetilde{T}}\right)$, see Definition 3.2. This yields that we may refer to functions $u_{T} \in B V\left(B^{n}, \mathcal{Y}\right)$ associated to currents $T$ in $\mathcal{E}_{1,1}-\operatorname{graph}\left(B^{n} \times \mathcal{Y}\right)$. 
JUMP-CONCENTRATION SET. If $\mathcal{L}(T)$ denotes the $(n-1)$-rectifiable set given by the union of the sets of positive multiplicity of the $\mathbb{L}_{s}(T)$ 's, we infer that the union

$$
J_{c}(T):=J_{u_{T}} \cup \mathcal{L}(T)
$$

does not depend on the choice of the representative in $T$. As in dimension one, the countably $\mathcal{H}^{n-1}$-rectifiable set $J_{c}(T)$ is said to be the set of points of jumpconcentration of $T$.

RESTRICTION OVER POINTS OF JUMP-CONCENTRATION. Let $T \in \mathcal{E}_{1,1}$ $-\operatorname{graph}\left(B^{n} \times \mathcal{Y}\right)$ and let $\nu_{T}: J_{c}(T) \rightarrow S^{n-1}$ denote an extension to $J_{c}(T)$ of the unit normal $\nu_{u_{T}}$ to the Jump set $J_{u_{T}}$. For any $k=1, \ldots, n-1$, let $P$ be an oriented $k$-dimensional subspace in $\mathbb{R}^{n}$ and $P_{\lambda}:=P+\sum_{i=1}^{n-k} \lambda_{i} \nu_{i}$ the family of oriented $k$-planes parallel to $P$, where $\lambda:=\left(\lambda_{1}, \ldots, \lambda_{n-k}\right) \in \mathbb{R}^{n-k}$, $\operatorname{span}\left(\nu_{1}, \ldots, \nu_{n-k}\right)$ being the orthogonal space to $P$. Since $T$ has finite $\mathcal{E}_{1,1}$-norm, similarly to the case of normal currents, for $\mathcal{L}^{n-k}$-a.e. $\lambda$ such that $P_{\lambda} \cap B^{n} \neq \emptyset$, the slice $T\left\llcorner\pi^{-1}\left(P_{\lambda}\right)\right.$ of $T$ over $\pi^{-1}\left(P_{\lambda}\right)$ is a well defined $k$-dimensional current in $\mathcal{E}_{1,1}-\operatorname{graph}\left(\left(B^{n} \cap P_{\lambda}\right) \times \mathcal{Y}\right)$ with finite $\mathcal{E}_{1,1}$-norm. Moreover, for any such $\lambda$ we have

$$
J_{c}\left(T\left\llcorner\pi^{-1}\left(P_{\lambda}\right)\right)=J_{c}(T) \cap P_{\lambda} \quad \text { in the } \mathcal{H}^{k-1}\right. \text {-a.e. sense, }
$$

whereas the $B V$-function associated to $T\left\llcorner P_{\lambda}\right.$ is equal to the restriction $u_{T \mid P_{\lambda}}$ of $u_{T}$ to $P_{\lambda}$. Therefore, in the particular case $k=1$, as in Sec. 1 the 1-dimensional restriction

$$
\widehat{\pi}_{\#}\left(\left(T\left\llcorner\pi^{-1}\left(P_{\lambda}\right)\right)\llcorner\{x\} \times \mathcal{Y}) \in \mathcal{D}_{1}(\mathcal{Y})\right.\right.
$$

of the 1-dimensional current $T\left\llcorner\pi^{-1}\left(P_{\lambda}\right)\right.$ over any point $x \in J_{c}(T) \cap P_{\lambda}$ such that $\nu_{T}(x)$ does not belong to $P$ is well defined. In this case, from the slicing properties of $B V$-functions, if $x \in\left(J_{c}(T) \backslash J_{u_{T}}\right) \cap P_{\lambda}$ we have $u_{T \mid P_{\lambda}}(x)=u_{T}(x)$. Moreover, if $x \in J_{u_{T}} \cap P_{\lambda}$, the one-sided approximate limits of $u_{T}$ are equal to the one-sided limits of the restriction $u_{T \mid P_{\lambda}}$, i.e.

$$
u_{T \mid P_{\lambda}}^{+}(x)=u_{T}^{+}(x) \quad \text { and } \quad u_{T \mid P_{\lambda}}^{-}(x)=u_{T}^{-}(x),
$$

provided that $\left\langle\nu, \nu_{u_{T}}(x)\right\rangle>0$, where $\nu$ is an orienting unit vector to $P$. We finally infer that, for $\mathcal{H}^{n-1}$-a.e. point $x \in J_{c}(T)$, the 1-dimensional restriction (3.8), up to the orientation, does not depend on the choice of the oriented 1-space $P$ and on $\lambda \in \mathbb{R}^{n-1}$, provided that $x \in P_{\lambda}$ and $\nu_{T}(x)$ does not belong to $P$. As a consequence we may and do give the following

Definition 3.5. For $\mathcal{H}^{n-1}$-a.e. point $x \in J_{c}(T)$, the 1-dimensional restriction $\widehat{\pi}_{\#}\left(T\llcorner\{x\} \times \mathcal{Y})\right.$ is given by (3.8) for any oriented 1 -space $P$ and $\lambda \in \mathbb{R}^{n-1}$ such that $x \in P_{\lambda}$ and $\left\langle\nu, \nu_{T}(x)\right\rangle>0$, where $\nu$ is the orienting unit vector to $P$. 
$B V$-ENERGY. The gap phenomenon and the properties previously described lead us to define the $B V$-energy of a current $T \in \mathcal{E}_{1,1}-\operatorname{graph}\left(B^{n} \times \mathcal{Y}\right)$ as follows.

Definition 3.6. For $\mathcal{H}^{n-1}$-a.e. point $x \in J_{c}(T)$ we define $\Gamma_{T}(x)$ and $\mathcal{L}_{T}(x)$ by (2.5) and (2.6), respectively, where $\widehat{\pi}_{\#}(T\llcorner\{x\} \times \mathcal{Y})$ is the 1-dimensional restriction given by Definition 3.5.

Definition 3.7. The $B V$-energy of a current $T \in \mathcal{E}_{1,1}-\operatorname{graph}\left(B^{n} \times \mathcal{Y}\right)$ is defined by

$$
\mathcal{E}_{1,1}(T, B \times \mathcal{Y}):=\int_{B}\left|\nabla u_{T}(x)\right| d x+\left|D^{C} u_{T}\right|(B)+\int_{J_{c}(T) \cap B} \mathcal{L}_{T}(x) d \mathcal{H}^{n-1}(x)
$$

for every Borel set $B \subset B^{n}$. We also let

$$
\mathcal{E}_{1,1}(T):=\mathcal{E}_{1,1}\left(T, B^{n} \times \mathcal{Y}\right) .
$$

Of course, if $T=G_{u}$ is the current integration of $n$-forms in $\mathcal{D}^{n, 1}\left(B^{n} \times \mathcal{Y}\right)$ over the graph of a function $u \in W^{1,1}\left(B^{n}, \mathcal{Y}\right)$, see $(1.8)$, then

$$
\mathcal{E}_{1,1}\left(G_{u}, B\right)=\int_{B}|D u(x)| d x
$$

Cartesian Currents. We then give the following

Definition 3.8. We denote by $\operatorname{cart}^{1,1}\left(B^{n} \times \mathcal{Y}\right)$ the class of currents $T$ in $\mathcal{E}_{1,1}-\operatorname{graph}\left(B^{n} \times \mathcal{Y}\right)$ such that $\mathcal{E}_{1,1}(T)<\infty$.

LOWER SEMICONTINUITY. Using the lower semicontinuity result in dimension $n=1$, see Theorem 2.6, and applying arguments as for instance in [4], we obtain in any dimension $n \geq 2$

Theorem 3.9. Let $T \in \operatorname{cart}^{1,1}\left(B^{n} \times \mathcal{Y}\right)$. For every sequence of smooth maps $\left\{u_{k}\right\} \subset C^{1}\left(B^{n}, \mathcal{Y}\right)$ such that $G_{u_{k}} \rightarrow T$ weakly in $\mathcal{Z}_{n, 1}\left(B^{n} \times \mathcal{Y}\right)$, we have

$$
\liminf _{k \rightarrow \infty} \int_{B^{n}}\left|D u_{k}\right| d x \geq \mathcal{E}_{1,1}(T) .
$$

A STRong DEnsity RESUlt. In [12] it is also proved in any dimension $n \geq 2$

Theorem 3.10. Assume that the first homotopy group $\pi_{1}(\mathcal{Y})$ is commutative. Let $T \in \operatorname{cart}^{1,1}\left(B^{n} \times \mathcal{Y}\right)$. There exists a sequence of smooth maps $\left\{u_{k}\right\} \subset$ $C^{1}\left(B^{n}, \mathcal{Y}\right)$ such that $G_{u_{k}} \rightarrow T$ weakly in $\mathcal{Z}_{n, 1}\left(B^{n} \times \mathcal{Y}\right)$ and $\left\|D u_{k}\right\|_{L^{1}} \rightarrow \mathcal{E}_{1,1}(T)$ as $k \rightarrow \infty$.

Remark 3.11. We notice that the commutativity hypothesis on the first homotopy group $\pi_{1}(\mathcal{Y})$ cannot be removed. If $\pi_{1}(\mathcal{Y})$ is non-abelian, even in dimension 
$n=2$ we find functions $u \in W^{1,1}\left(B^{2}, \mathcal{Y}\right)$, smooth outside the origin and satisfying the null-boundary condition

$$
\partial G_{u}=0 \quad \text { on } \quad \mathcal{Z}^{n-1,1}\left(B^{n} \times \mathcal{Y}\right),
$$

such that for every sequence of smooth maps $u_{k}: B^{n} \rightarrow \mathcal{Y}$ for which $G_{u_{k}} \rightarrow G_{u}$ weakly in $\mathcal{Z}_{n, 1}\left(B^{n} \times \mathcal{Y}\right)$ we have

$$
\liminf _{k \rightarrow \infty} \int_{B^{2}}\left|D u_{k}\right| d x \geq C+\int_{B^{2}}|D u| d x
$$

for some absolute constant $C>0$. For example, compare [11] in the case of the area.

RELAXED TOTAL VARIATION FUNCTIONAL. In the sequel we shall always assume that the first homotopy group $\pi_{1}(\mathcal{Y})$ is commutative. As a consequence, setting

$$
\widetilde{\mathcal{E}_{1,1}}(T):=\inf \left\{\liminf _{k \rightarrow \infty} \int_{B^{n}}\left|D u_{k}\right| d x:\left\{u_{k}\right\} \subset C^{1}\left(B^{n}, \mathcal{Y}\right), \quad G_{u_{k}} \rightarrow T\right.
$$

weakly in $\left.\mathcal{Z}_{n, 1}\left(B^{n} \times \mathcal{Y}\right)\right\}$, by Theorems 3.9 and 3.10 we conclude that

$$
\mathcal{E}_{1,1}(T)=\widetilde{\mathcal{E}_{1,1}}(T) \quad \forall T \in \operatorname{cart}^{1,1}\left(B^{n} \times \mathcal{Y}\right) .
$$

Properties. By Theorems 3.9 and 3.10 we readily infer the following lower semicontinuity result.

Proposition 3.12. Let $\left\{T_{k}\right\} \subset \operatorname{cart}^{1,1}\left(B^{n} \times \mathcal{Y}\right)$ converge weakly in $\mathcal{Z}_{n, 1}\left(B^{n} \times \mathcal{Y}\right)$, $T_{k} \rightarrow T$, to some $T \in \operatorname{cart}^{1,1}\left(B^{n} \times \mathcal{Y}\right)$. Then

$$
\mathcal{E}_{1,1}(T) \leq \liminf _{k \rightarrow \infty} \mathcal{E}_{1,1}\left(T_{k}\right)
$$

Moreover, we obtain that the class of Cartesian currents $\operatorname{cart}^{1,1}\left(B^{n} \times \mathcal{Y}\right)$ is closed under weak convergence with equibounded energies.

Theorem 3.13. Let $\left\{T_{k}\right\} \subset \operatorname{cart}^{1,1}\left(B^{n} \times \mathcal{Y}\right)$ converge weakly in $\mathcal{Z}_{n, 1}\left(B^{n} \times \mathcal{Y}\right)$, $T_{k} \rightarrow T$, to some $T \in \mathcal{D}_{n, 1}\left(B^{n} \times \mathcal{Y}\right)$, and $\sup _{k} \mathcal{E}_{1,1}\left(T_{k}\right)<\infty$. Then $T \in$ $\operatorname{cart}^{1,1}\left(B^{n} \times \mathcal{Y}\right)$.

Therefore, by the relative compactness of $\mathcal{E}_{1,1}$-bounded sets in $\mathcal{D}_{n, 1}\left(B^{n} \times \mathcal{Y}\right)$ we readily infer the following compactness property.

Proposition 3.14. Let $\left\{T_{k}\right\} \subset \operatorname{cart}^{1,1}\left(B^{n} \times \mathcal{Y}\right)$ be such that $\sup _{k} \mathcal{E}_{1,1}\left(T_{k}\right)<\infty$. Then, possibly passing to a subsequence, $T_{k} \rightarrow T$ weakly in $\mathcal{Z}_{n, 1}\left(B^{n} \times \mathcal{Y}\right)$ to some $T \in \operatorname{cart}^{1,1}\left(B^{n} \times \mathcal{Y}\right)$. 
Example 3.15. Of course, for every $T \in \operatorname{cart}^{1,1}\left(B^{n} \times \mathcal{Y}\right)$ we may decompose

$$
T=T_{u}+S_{T} \quad \text { on } \quad \mathcal{Z}^{n, 1}\left(B^{n} \times \mathcal{Y}\right),
$$

where $u=u_{T} \in B V\left(B^{n}, \mathcal{Y}\right)$ is the $B V$-function corresponding to $T$ and $T_{u} \in$ $B V-\operatorname{graph}\left(B^{n} \times \mathcal{Y}\right)$ is defined as in Sec. 1 above, i.e., by means of geodesic arcs $l_{x}$ connecting $u^{-}(x)$ and $u^{+}(x)$ at the points $x$ in the jump set $J_{u}$. However, even in dimension $n=1$ and in the particular case $\mathcal{Y}=S^{1}$, the unit sphere, in general it may happen that the $B V$-energy of $T$ cannot be recovered by the sum of the $B V$-energies of its component $T_{u}$ and $S_{T}$ in (3.10). If $\mathcal{Y}=S^{1}$, in fact, we have $S_{T, s i n g}=0$, i.e., the equivalence classes of elements in cart ${ }^{1,1}\left(B^{n} \times S^{1}\right)$ have a unique representative, and the energies $\mathcal{E}_{1,1}(T)$ and $\mathcal{F}_{1,1}(T)$ are equal, i.e., no gap phenomenon occurs. Consider the current $T^{\theta} \in \operatorname{cart}^{1,1}\left(B^{1} \times S^{1}\right)$ given by

$$
T^{\theta}=\llbracket(-1,0) \rrbracket \times \delta_{P_{0}}+\llbracket(0,1) \rrbracket \times \delta_{P_{\theta}}+\delta_{0} \times \gamma_{\theta}, \quad \theta \in[0,2 \pi],
$$

where $P_{\theta}=(\cos \theta, \sin \theta)$ and $\gamma_{\theta}$ is the simple arc in $S^{1}$ connecting the points $P_{0}$ and $P_{\theta}$ in the counterclockwise sense. If $\pi<\theta<2 \pi$ we clearly have

$$
T_{u}=\llbracket(-1,0) \rrbracket \times \delta_{P_{0}}+\llbracket(0,1) \rrbracket \times \delta_{P_{\theta}}+\delta_{0} \times \widetilde{\gamma}_{\theta},
$$

where $\widetilde{\gamma}_{\theta}$ is the simple arc in $S^{1}$ connecting the points $P_{0}$ and $P_{\theta}$ in the clockwise sense, so that we may decompose $T^{\theta}$ as in (3.10) with $S_{T}=\delta_{0} \times \llbracket S^{1} \rrbracket$. Since

$$
\mathcal{F}_{1,1}\left(T_{u}\right)=\mathcal{H}^{1}\left(\widetilde{\gamma}_{\theta}\right)=2 \pi-\theta, \quad \mathcal{F}_{1,1}\left(S_{T}\right)=2 \pi,
$$

we infer that the sum of the energies $\mathcal{F}_{1,1}\left(T_{u}\right)+\mathcal{F}_{1,1}\left(S_{T}\right)$ is greater than the energy of $T^{\theta}$, as clearly

$$
\mathcal{E}_{1,1}\left(T^{\theta}\right)=\mathcal{F}_{1,1}\left(T^{\theta}\right)=\mathcal{H}^{1}\left(\gamma_{\theta}\right)=\theta .
$$

\section{The Relaxed BV-energy of FunCtions}

In this section we analyze the relaxed $B V$-energy $\widetilde{\mathcal{E}_{T V}}(u)$ of functions $u$ in $B V\left(B^{n}, \mathcal{Y}\right)$, i.e., the lower semicontinuous envelope of the total variation functional (1.3). Of course, it may be equivalently defined for every function $u \in B V\left(B^{n}, \mathcal{Y}\right)$ by

$$
\widetilde{\mathcal{E}_{T V}}(u):=\inf \left\{\liminf _{k \rightarrow \infty} \int_{B^{n}}\left|D u_{k}\right| d x \mid\left\{u_{k}\right\} \subset C^{1}\left(B^{n}, \mathcal{Y}\right), \quad u_{k} \rightarrow u\right.
$$

weakly in the $B V$-sense $\}$.

Remark 4.1. It is obvious that one may equivalently require that $u_{k} \rightarrow u$ strongly in the $L^{1}$-sense. 
In the sequel we assume that the first homotopy group $\pi_{1}(\mathcal{Y})$ is commutative. Moreover, we denote by

$$
\mathcal{T}_{u}:=\left\{T \in \operatorname{cart}^{1,1}\left(B^{n}, \mathcal{Y}\right) \mid u_{T}=u\right\}
$$

the class of Cartesian currents $T$ in $\operatorname{cart}^{1,1}\left(B^{n} \times \mathcal{Y}\right)$ such that the underlying $B V$-function $u_{T}$ is equal to $u$, compare Definition 3.8 and Remark 3.4.

For future use, we also recall from [9], Vol. II, Sec. 5.4.2, that for every $u \in$ $W^{1,1}\left(B^{n}, \mathcal{Y}\right)$ the homological singularities of $u$ are well-defined for every $s=$ $1, \ldots, \bar{s}$ by the currents $\mathbb{P}_{s}(u) \in \mathcal{D}_{n-2}\left(B^{n}\right)$ given by

$$
\mathbb{P}_{s}(u)(\phi):=\partial G_{u}\left(\pi^{\#} \phi \wedge \widehat{\pi}^{\#} \omega^{s}\right) \quad \forall \phi \in \mathcal{D}^{n-2}\left(B^{n}\right) .
$$

We finally recall the following facts.

Definition 4.2. For every $k=2, \ldots, n$ and $\Gamma \in \mathcal{D}_{n-k}\left(B^{n}\right)$, we denote by

$$
m_{i, B^{n}}(\Gamma):=\inf \left\{\mathbf{M}(L) \mid L \in \mathcal{R}_{n-k+1}\left(B^{n}\right), \quad(\partial L)\left\llcorner B^{n}=\Gamma\right\}\right.
$$

the integral mass of $\Gamma$ and by

$$
m_{r, B^{n}}(\Gamma):=\inf \left\{\mathbf{M}(D) \mid D \in \mathcal{D}_{n-k+1}\left(B^{n}\right),(\partial D)\left\llcorner B^{n}=\Gamma\right\}\right.
$$

the real mass of $\Gamma$. Moreover, in case $m_{i, B^{n}}(\Gamma)<\infty$, we say that an i.m. rectifiable current $L \in \mathcal{R}_{n-k+1}\left(B^{n}\right)$ is an integral minimal connection of $\Gamma$ if $(\partial L)\left\llcorner B^{n}=\Gamma\right.$ and $\mathbf{M}(L)=m_{i, B^{n}}(\Gamma)$.

By Federer's theorem [7], and by Hardt-Pitts' theorem [13], respectively, in the cases $k=n$ and $k=2$ we have that

$$
m_{i, B^{n}}(\Gamma)=m_{r, B^{n}}(\Gamma) .
$$

RESUlTs. We have the following.

Theorem 4.3. For every $u \in B V\left(B^{n}, \mathcal{Y}\right)$ we have $\widetilde{\mathcal{E}_{T V}}(u)<\infty$.

From the results of the previous sections, we then obtain the following representation.

Theorem 4.4. For any $u \in B V\left(B^{n}, \mathcal{Y}\right)$ we have

$$
\begin{aligned}
\widetilde{\mathcal{E}_{T V}}(u) & =\inf \left\{\mathcal{E}_{1,1}(T) \mid T \in \mathcal{T}_{u}\right\} \\
& =\int_{B^{n}}|\nabla u(x)| d x+\left|D^{C} u\right|\left(B^{n}\right)+\inf \left\{\int_{J_{c}(T)} \mathcal{L}_{T}(x) d \mathcal{H}^{n-1}(x) \mid T \in \mathcal{T}_{u}\right\},
\end{aligned}
$$

where $\mathcal{T}_{u}, J_{c}(T)$, and $\mathcal{L}_{T}(x)$ are given by (4.1), (3.7), and Definition 3.6, respectively. 
Sketch of The Proof of Theorem 4.3: We first notice that it suffices to show for every $u \in B V\left(B^{n}, \mathcal{Y}\right)$ the class $\mathcal{T}_{u}$ is non-empty, see (4.1). In this case, in fact, if $T \in \mathcal{T}_{u}$, by Theorem 3.10 we find a smooth sequence $\left\{u_{k}\right\} \subset C^{1}\left(B^{n}, \mathcal{Y}\right)$ such that $G_{u_{k}} \rightarrow T$ weakly in $\mathcal{Z}_{n, 1}\left(B^{n} \times \mathcal{Y}\right)$ and $\left\|D u_{k}\right\|_{L^{1}} \rightarrow \mathcal{E}_{1,1}(T)$ as $k \rightarrow \infty$; this yields also that $u_{k} \rightarrow u_{T}$ weakly in the $B V$-sense, where $u_{T}=u$.

Now, if $\left\{u_{k}\right\}$ is the approximating sequence given by Proposition 1.8, since $u_{k} \in R_{1}^{\infty}\left(B^{n}, \mathcal{Y}\right)$, the real mass of the singularities $\mathbb{P}_{s}\left(u_{k}\right)$, see (4.2), is bounded by the $L^{1}$-norm of $D u_{k}$. Moreover, by Hardt-Pitts' theorem (4.3) we infer that the real mass agrees with the integral mass. Therefore, we are able to connect the singularities of the $u_{k}$ 's by means of i.m. rectifiable currents in $\mathcal{R}_{n-1}\left(B^{n}\right)$, this way defining a sequence

$$
T_{k}:=G_{u_{k}}+\sum_{s=1}^{\bar{s}} \mathbb{L}_{s}^{k} \times \gamma_{s} \in \operatorname{cart}^{1,1}\left(B^{n} \times \mathcal{Y}\right),
$$

where $\mathbb{L}_{s}^{k} \in \mathcal{R}_{n-1}\left(B^{n}\right)$ is such that

$$
\mathbb{P}_{s}\left(u_{k}\right)=-\left(\partial \mathbb{L}_{s}^{k}\right)\left\llcorner B^{n} \quad \text { and } \quad \mathbf{M}\left(\mathbb{L}_{s}^{k}\right) \leq C \int_{B^{n}}\left|D u_{k}\right| d x,\right.
$$

so that

$$
\sup _{k} \mathcal{E}_{1,1}\left(T_{k}\right) \leq C \sup _{k} \int_{B^{n}}\left|D u_{k}\right| d x \leq C \mathcal{E}_{T V}\left(u, B^{n}\right)<\infty,
$$

where $C>0$ is an absolute constant. Therefore, by compactness, Proposition 3.14, possibly passing to a subsequence we find that $T_{k} \rightarrow T$ weakly in $\mathcal{Z}_{n, 1}\left(B^{n} \times \mathcal{Y}\right)$ to some $T \in \operatorname{cart}^{1,1}\left(B^{n} \times \mathcal{Y}\right)$ satisfying

$$
\mathcal{E}_{1,1}(T) \leq \liminf _{k \rightarrow \infty} \mathcal{E}_{1,1}\left(T_{k}\right)<\infty
$$

by lower semicontinuity, Proposition 3.12. In particular, since $u_{k} \rightarrow u$ weakly in the $B V$-sense, we find that the underlying $B V$-function $u_{T}=u$ and hence that $T \in \mathcal{T}_{u}$, as required.

Proof of TheOREM 4.4: Let $\left\{u_{k}\right\} \subset C^{1}\left(B^{n}, \mathcal{Y}\right)$ be a smooth sequence with equibounded energies, $\sup _{k}\left\|D u_{k}\right\|_{L^{1}}<\infty$, and weakly converging to $u$ in the $B V$-sense, see Theorem 4.3. By compactness, Proposition 3.14, possibly passing to a subsequence we find that $G_{u_{k}} \rightarrow T$ weakly in $\mathcal{Z}_{n, 1}\left(B^{n} \times \mathcal{Y}\right)$ to some $T \in$ $\operatorname{cart}^{1,1}\left(B^{n} \times \mathcal{Y}\right)$ satisfying $u_{T}=u$, i.e., $T \in \mathcal{T}_{u}$, see (4.1). Since by lower semicontinuity, Proposition 3.12,

$$
\mathcal{E}_{1,1}(T) \leq \liminf _{k \rightarrow \infty} \int_{B^{n}}\left|D u_{k}\right| d x,
$$

we readily conclude that

$$
\inf \left\{\mathcal{E}_{1,1}(T) \mid T \in \mathcal{T}_{u}\right\} \leq \widetilde{\mathcal{E}_{T V}}(u) .
$$


To prove the opposite inequality, by applying Theorem 3.10, for every $T \in \mathcal{T}_{u}$ we find a sequence of smooth maps $\left\{u_{k}\right\} \subset C^{1}\left(B^{n}, \mathcal{Y}\right)$ such that $G_{u_{k}} \rightarrow T$ weakly in $\mathcal{Z}_{n, 1}\left(B^{n} \times \mathcal{Y}\right)$ and $\left\|D u_{k}\right\|_{L^{1}} \rightarrow \mathcal{E}_{1,1}(T)$ as $k \rightarrow \infty$. Since the weak convergence $G_{u_{k}} \rightarrow T$ yields the convergence $u_{k} \rightarrow u_{T}$ weakly in the $B V$-sense, and $u_{T}=u$, we find that $\widetilde{\mathcal{E}_{T V}}(u) \leq \mathcal{E}_{1,1}(T)$, which proves the first equality in (4.4). The second equality in (4.4) follows from the definition of $B V$-energy, Definition 3.7.

The above facts simplify if we specify them to $u \in W^{1,1}\left(B^{n}, \mathcal{Y}\right)$ and/or $\mathcal{Y}=$ $S^{1}$, recovering this way previous results, compare e.g. [8], [5], and [14].

The RELAXED $W^{1,1}$-ENERGY. The relaxed energy of $u \in W^{1,1}\left(B^{n}, \mathcal{Y}\right)$ is of course given by

$$
\widetilde{\mathcal{E}_{1,1}}(u):=\inf \left\{\liminf _{k \rightarrow \infty} \int_{B^{n}}\left|D u_{k}\right| d x \quad \mid \quad\left\{u_{k}\right\} \subset C^{1}\left(B^{n}, \mathcal{Y}\right), \quad u_{k} \rightarrow u\right.
$$

strongly in $\left.L^{1}\left(B^{n}, \mathbb{R}^{N}\right)\right\}$,

see Remark 4.1. In this case, Theorem 4.4 reads as

Corollary 4.5. For any $u \in W^{1,1}\left(B^{n}, \mathcal{Y}\right)$ we have $\widetilde{\mathcal{E}_{1,1}}(u)<\infty$. Every $T \in \mathcal{T}_{u}$ has the form

$$
T=G_{u}+\sum_{q \in H_{1}(\mathcal{Y})} \mathbb{L}_{q} \times C_{q} \quad \text { on } \quad \mathcal{Z}^{n, 1}\left(B^{n} \times \mathcal{Y}\right),
$$

where $\mathbb{L}_{q}=\tau\left(\mathcal{L}_{q}, 1, \overrightarrow{\mathcal{L}}_{q}\right)$, see [6], is an i.m. rectifiable current in $\mathcal{R}_{n-1}\left(B^{n}\right)$, and $C_{q} \in \mathcal{Z}_{1}(\mathcal{Y})$ is an integral 1-cycle in the homology class $q$. The $B V$-energy of $T$ is given by

$$
\mathcal{E}_{1,1}(T)=\int_{B^{n}}|D u| d x+\sum_{q \in H_{1}(\mathcal{Y})} \int_{\mathcal{L}_{q}} \mathcal{L}_{T}(x) d \mathcal{H}^{n-1}(x)
$$

where, for $x \in \mathcal{L}_{q}$, we have $\mathcal{L}_{T}(x):=\inf \left\{\mathcal{L}(\gamma) \mid \gamma \in \Gamma_{q}(x)\right\}$ and

$$
\Gamma_{q}(x):=\left\{\gamma \in \operatorname{Lip}([0,1], \mathcal{Y}) \mid \gamma(0)=\gamma(1)=u(x), \quad \gamma_{\#} \llbracket(0,1) \rrbracket \in q\right\} .
$$

Finally, the relaxed energy of $u$ is given by

$$
\widetilde{\mathcal{E}_{1,1}}(u)=\int_{B^{n}}|D u(x)| d x+\inf \left\{\sum_{q \in H_{1}(\mathcal{Y})} \int_{\mathcal{L}_{q}} \mathcal{L}_{T}(x) d \mathcal{H}^{n-1}(x) \mid T \in \mathcal{T}_{u}\right\} .
$$

THE CASE $\mathcal{Y}=S^{1}$. Further simplification arises if we assume $\mathcal{Y}=S^{1}$. In this case, in fact, $S_{T, s i n g}=0$, i.e. the equivalence classes of elements in $\operatorname{cart}^{1,1}\left(B^{n} \times S^{1}\right)$ have a unique representative, and the energies $\mathcal{E}_{1,1}(T)$ and 
$\mathcal{F}_{1,1}(T)$ are equal, i.e., no gap phenomenon occurs. Moreover, if $x$ belongs to the jump-concentration set $J_{c}(T)$, the 1-dimensional restriction has the form

$$
\widehat{\pi}_{\#}\left(T\left\llcorner\{x\} \times S^{1}\right)=\llbracket \gamma_{x} \rrbracket+q \llbracket S^{1} \rrbracket,\right.
$$

where $q \in \mathbb{Z}$ and $\llbracket \gamma_{x} \rrbracket$ is the current associated to a suitably oriented simple arc $\gamma_{x}$ in $S^{1}$ connecting the points $u_{T}^{-}(x)$ and $u_{T}^{+}(x)$, where $u_{T}$ is the function in $B V\left(B^{n}, S^{1}\right)$ associated to $T$, and $\gamma_{x}=0$ if $x \notin J_{u_{T}}$. Consequently, in (4.4) we have

$$
\mathcal{L}_{T}(x)=\mathcal{H}^{1}\left(\gamma_{x}\right)+2 \pi|q|
$$

and hence for every $T \in \operatorname{cart}^{1,1}\left(B^{n} \times S^{1}\right)$ the $B V$-energy agrees with the energy obtained in [8], compare Theorem 1 of [9, Vol. II, Sec. 6.2.3]. Moreover, we recover the following estimates about the relaxed energy, compare [5] and [14].

Proposition 4.6. For every $u \in W^{1,1}\left(B^{n}, S^{1}\right)$ we have

$$
\widetilde{\mathcal{E}_{1,1}}(u) \leq 2 \mathcal{E}_{1,1}(u), \quad \text { where } \quad \mathcal{E}_{1,1}(u):=\int_{B^{n}}|D u| d x .
$$

Moreover, for every $u \in B V\left(B^{n}, S^{1}\right)$ we have

$$
\widetilde{\mathcal{E}_{T V}}(u) \leq 2 \mathcal{E}_{T V}(u),
$$

where $\mathcal{E}_{T V}(u)$ is the total variation of $u$, given by (1.1).

Proof. If $u \in W^{1,1}\left(B^{n}, S^{1}\right)$, its singular set is the current $\mathbb{P}(u) \in \mathcal{D}_{n-2}\left(B^{n}\right)$ given for any $\phi \in \mathcal{D}^{n-2}\left(B^{n}\right)$ by $\mathbb{P}(u)(\phi):=\partial G_{u}\left(\pi^{\#} \phi \wedge \widehat{\pi}^{\#} \omega_{S^{1}}\right)$, where

$$
\omega_{S^{1}}:=\frac{1}{2 \pi}\left(y^{1} d y^{2}-y^{2} d y^{1}\right)
$$

is the normalized volume 1 -form in $S^{1}$. Therefore, $\mathbb{P}(u)$ is the boundary of the current $\mathbb{D}(u) \in \mathcal{D}_{n-1}\left(B^{n}\right)$ defined for any $\phi \in \mathcal{D}^{n-1}\left(B^{n}\right)$ by $\mathbb{D}(u)(\phi):=$ $G_{u}\left(\pi^{\#} \phi \wedge \widehat{\pi}^{\#} \omega_{S^{1}}\right)$. Since we estimate

$$
\mathbf{M}(\mathbb{D}(u)) \leq \frac{1}{2 \pi} \int_{B^{n}}|D u| d x,
$$

we infer that the real mass $m_{r, B^{n}}(\mathbb{P}(u)) \leq \mathcal{E}_{1,1}\left(u, B^{n}\right) / 2 \pi$ and therefore, by Hardt-Pitts' theorem [13], that the integral mass $m_{i, B^{n}}(\mathbb{P}(u)) \leq \mathcal{E}_{1,1}\left(u, B^{n}\right) / 2 \pi$, see Definition 4.2 and (4.3). As a consequence, since for every $\varepsilon>0$ we find a current $T \in \mathcal{T}_{u}$ such that

$$
T=G_{u}+L \times S^{1} \quad \text { and } \quad \mathcal{E}_{1,1}(T)=\mathcal{E}_{1,1}(u)+2 \pi \mathbf{M}(L),
$$

where $L \in \mathcal{R}_{n-1}\left(B^{n}\right)$ satisfies $\mathbf{M}(L) \leq m_{i, B^{n}}(\mathbb{P}(u))+\varepsilon$, taking into account Theorem 4.4 we obtain (4.5).

In the more general case $u \in B V\left(B^{n}, S^{1}\right)$, by Proposition 1.8 we find the existence of a sequence of maps $\left\{u_{k}\right\} \subset W^{1,1}\left(B^{n}, S^{1}\right)$ such that $u_{k} \rightarrow u$ weakly in the $B V$-sense and $\mathcal{E}_{1,1}\left(u_{k}\right) \rightarrow \mathcal{E}_{T V}(u)$. Moreover, for every $k$ we find a smooth 
sequence $\left\{u_{h}^{(k)}\right\}_{h} \subset C^{1}\left(B^{n}, S^{1}\right)$ converging to $u_{k}$ strongly in $L^{1}$ and such that $\mathcal{E}_{1,1}\left(u_{h}^{(k)}\right) \rightarrow \widehat{\mathcal{E}_{1,1}}\left(u_{k}\right)+1 / k$ as $h \rightarrow \infty$. Finally, by (4.5) and by a diagonal argument we readily obtain (4.6).

Remark 4.7. Following [15], since $\pi_{1}(\mathcal{Y})$ is commutative, if $u \in R_{1}^{\infty}\left(B^{n}, \mathcal{Y}\right)$, for every $s=1, \ldots, \bar{s}$ we may find an integral current $L_{s} \in \mathcal{R}_{n-2}\left(B^{n}\right)$ satisfying

$$
-\left(\partial L_{s}\right)\left\llcorner B^{n}=\mathbb{P}_{s}(u) \quad \text { and } \quad \mathbf{M}\left(L_{s}\right) \leq C \int_{B^{n}}|D u| d x,\right.
$$

see (4.2), where the absolute constant $C>0$ does not depend on $u$. Therefore, arguing as above it is not difficult to show that for every $u \in W^{1,1}\left(B^{n}, \mathcal{Y}\right)$

$$
\widetilde{\mathcal{E}_{1,1}}(u) \leq C(n, \mathcal{Y}) \cdot \mathcal{E}_{1,1}(u),
$$

where $C(n, \mathcal{Y})>0$ is an absolute constant, only depending on $n$ and $\mathcal{Y}$. Finally, by Proposition 1.8, we conclude that

$$
\widetilde{\mathcal{E}_{T V}}(u) \leq C(n, \mathcal{Y}) \cdot \mathcal{E}_{T V}(u) \quad \forall u \in B V\left(B^{n}, \mathcal{Y}\right),
$$

where $\mathcal{E}_{T V}(u)$ is the total variation of $u$ given by (1.1) and the optimal constant $C(n, \mathcal{Y})$ is the same as the optimal constant for $W^{1,1}$-functions in (4.7).

Acknowledgement. The second author thanks the Research Center Ennio De Giorgi of the Scuola Normale Superiore of Pisa for the hospitality during the preparation of this paper.

\section{REFERENCES}

[1] AMBROSIO L., Metric space valued functions of bounded variation. Ann. Scuola Norm. Sup. Pisa Cl. Sci. 17 (1990) 439-478.

[2] AMBROSIO L., FUSCO N., PALLARA D., Functions of bounded variation and free discontinuity problems. Oxford Math. Monographs, Oxford (2000).

[3] BETHUEL F., The approximation problem for Sobolev maps between manifolds. Acta Math. 167 (1992) 153-206.

[4] BOUChitTÉ G., BUTTAZZO G., New lower semicontinuity results for nonconvex functionals defined on measures. Nonlinear Anal. 15 (1990) 679-692.

[5] BREZIS H., MIRONESCU P., PONCE A., $W^{1,1}$-maps with value into $S^{1}$. Geometric Analysis of PDE and Several Complex Variables (S. Chanillo, P. Cordaro, N. Hanges and A. Meziani, eds.), Contemporary Mathematics, 368. American Mathematical Society, Providence, RI (2005) 69-100.

[6] FEDERER H., Geometric measure theory. Grundlehren math. Wissen. 153, Springer, Berlin (1969).

[7] FEDERER H., Real flat chains, cochains and variational problems. Indiana Univ. Math. J. 24 (1974) 351-407.

[8] GIAQUINTA M., MODICA G., SOUČEK J., Variational problems for maps of bounded variations with values in $S^{1}$. Calc. Var. 1 (1993) 87-121.

[9] GIAQUINTA M., MODICA G., SOUČEK J., Cartesian currents in the calculus of variations, I, II. Ergebnisse Math. Grenzgebiete (III Ser), 37, 38, Springer, Berlin (1998). 
[10] GIAQUINTA M., MUCCI D., Weak and strong density results for the Dirichlet energy. $J$. Eur. Math. Soc. 6 (2004) 95-117.

[11] GIAQUINTA M., MUCCI D., The Dirichlet energy of mappings from $B^{3}$ into a manifold: density results and gap phenomenon. Calc. Var. 20 (2004) 367-397.

[12] M. GIAQUINTA, D. MUCCI, The $B V$-energy of maps into a manifold: relaxation and density results. Ann. Scuola Norm. Sup. Pisa Cl. Sci. (5) 5 (2006), 483-548.

[13] HARDT B., PITTS J., Solving the Plateau's problem for hypersurfaces without the compactness theorem for integral currents. In Geometric Measure Theory and the Calculus of Variations, edited by Allard W.K. and Almgren F.J.. Proc. Symp. Pure Math. 44. Am. Math. Soc., Providence (1996) 255-295.

[14] IGNAT R., The space $B V\left(S^{2} ; S^{1}\right)$ : minimal connections and optimal liftings. Annales de l'Institut Henri Poincaré. Analyse Non Linéaire 22 n. 3 (2005) 283-302.

[15] PAKZAD M.R., RIVIÈRE T., Weak density of smooth maps for the Dirichlet energy between manifolds. Geom. Funct. Anal. 13 (2001) 223-257.

[16] SIMON L., Lectures on geometric measure theory. Proc. C.M.A., Vol 3, Australian Natl. Univ., Canberra (1983).

Mariano Giaquinta

Scuola Normale Superiore

Piazza dei Cavalieri 7, I-56100 Pisa

E-mail: giaquinta@sns.it

Domenico Mucci

Dipartimento di Matematica dell'Università di Parma

Viale G. P. Usberti 53/A, I-43100 Parma

E-mail: domenico.mucci@unipr.it 